\title{
Identification and Quantification of Novel Major Metabolites of the Steroidal Aromatase Inhibitor, Exemestane
}

\author{
Shaman Luo, ${ }^{1}$ @ Gang Chen, ${ }^{1}$ Cristina I. Truica, Cynthia C. Baird, Zuping Xia, and Philip Lazarus
}

Department of Pharmaceutical Sciences, College of Pharmacy and Pharmaceutical Sciences, Washington State University, Spokane, Washington (S.L., G.C., Z.X., P.L.); Department of Medicine, Penn State University College of Medicine, Hershey, Pennsylvania (C.I.T., C.C.B.); and Alkali Soil Natural Environmental Science Center, Northeast Forestry University, Harbin, Heilongjiang, China (S.L.)

Received February 28, 2018; accepted September 14, 2018

\begin{abstract}
Exemestane (EXE) is an aromatase inhibitor used for the prevention and treatment of estrogen receptor-positive breast cancer. Although the known major metabolic pathway for EXE is reduction to form the active $17 \beta$-dihydro-EXE (17 $\beta$-DHE) and subsequent glucuronidation to $17 \beta$-hydroxy-EXE-17-O- $\beta$-D-glucuronide (17 $\beta$ DHE-Gluc), previous studies have suggested that other major metabolites exist for exemestane. In the present study, a liquid chromatography-mass spectrometry (LC-MS) approach was used to acquire accurate mass data in $\mathrm{MS}^{\mathrm{E}}$ mode, in which precursor ion and fragment ion data were obtained simultaneously to screen novel phase II EXE metabolites in urine specimens from women taking EXE. Two major metabolites predicted to be cysteine conjugates of EXE and $17 \beta$-DHE by elemental composition were identified. The structures of the two metabolites were confirmed to be
\end{abstract}

6-methylcysteinylandrosta-1,4-diene-3,17-dione (6-EXE-cys) and 6-methylcysteinylandrosta-1,4-diene-17 $\beta$-hydroxy-3-one (6-17 $\beta$ DHE-cys) after comparison with their chemically synthesized counterparts. Both underwent biosynthesis in vitro in three stepwise enzymatic reactions, with the first involving glutathione conjugation. The cysteine conjugates of EXE and 17 $\beta$-DHE were subsequently quantified by liquid chromatography-mass spectrometry in the urine and matched plasma samples of 132 subjects taking EXE. The combined 6-EXE-cys plus 6-17 $\beta$-DHE-cys made up $77 \%$ of total EXE metabolites in urine (vs. 1.7\%, $0.14 \%$, and $21 \%$ for EXE, $17 \beta$-DHE, and $17 \beta$-DHE-Gluc, respectively) and $35 \%$ in plasma (vs. $17 \%, 12 \%$, and $36 \%$ for EXE, $17 \beta$-DHE, and $17 \beta$-DHE-Gluc, respectively). Therefore, cysteine conjugates of EXE and 17 $\beta$-DHE appear to be major metabolites of EXE in both urine and plasma.

\section{Introduction}

Breast cancer is the most frequently diagnosed cancer in the United States (Howlader et al., 2016). An estimated 252,710 new cases of female breast cancer were diagnosed in the United States in 2017 with approximately $75 \%$ of these being estrogen receptor-positive (ER+) (Osborne and Schiff, 2011; Siegel et al., 2017). In postmenopausal women, treatment of early-stage ER+ breast cancer has focused primarily on the elimination of estrogen-induced tumor cell growth. Aromatase inhibitors (AIs), similar to the steroidal substrate analog exemestane (EXE), act to inhibit aromatase activity by blocking the biosynthesis of estrone and estradiol, thereby preventing estrogen-induced tumor cell growth (Miller, 1999; Campos, 2004; Santen et al., 2009). Clinical trials have shown

This work was supported by the National Institutes of Health National Cancer Institute [Grant R01-CA164366] to P.L., a China Scholarship Council grant for S.L. and a grant from the Health Sciences and Services Authority of Spokane, WA [Grant WSU002292] to WSU College of Pharmacy and Pharmaceutical Sciences.

${ }^{1}$ S.L. and G.C. contributed equally to this work.

There are no competing financial interests or conflicts of interest for any of the authors of this manuscript.

https://doi.org/10.1124/dmd.118.081166. that the use of AIs increases disease-free survival and decreases the occurrence of contralateral breast cancer as compared with the use of selective estrogen receptor modulators, such as tamoxifen (Howell et al., 2005; Ferretti et al., 2006; Eisen et al., 2008; Forbes et al., 2008). EXE is widely used for the adjuvant treatment and prevention of breast cancer in postmenopausal women (Wang and Chen, 2006; Hong et al., 2007; Deeks and Scott, 2009; Petkov et al., 2009). Although AIs such as EXE represent an improvement in treatment and prevention of breast cancer, considerable interindividual variation exists in patients' responses to these drugs (Paridaens et al., 2003; Chia et al., 2008; Campos et al., 2009; Glück, 2010). The causes for this interindividual variability have not been clearly elucidated.

Phase I metabolites of EXE have been identified in vivo. In addition to the major active phase I metabolite $17 \beta$-dihydro-EXE (17 $\beta$-DHE), several minor metabolites with much lower activities are formed, including $6 \xi$-hydroxy- $6 \xi$-hydroxymethylandrosta-1,4-diene-3,17-dione; $6 \xi$-hydroxyandrosta-1,4-diene-3,17-dione; $3 \xi$-hydroxy-5 $\xi$-androst1-ene-6-methylene-17-one; $6 \xi$-17 $\beta$-dihydroxy- $6 \xi$-hydroxymethylandrosta-

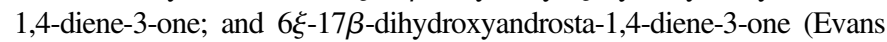
et al., 1992; Cavalcanti Gde et al., 2011; de Albuquerque Cavalcanti et al., 2011). Cytosolic aldo-keto reductase 1Cs and carbonyl reductase 1 are

ABBREVIATIONS: Al, aromatase inhibitor; AZA, azathioprine; $\mathrm{D}_{3}-17 \beta$-DHE, $17 \beta$-OH-EXE-d3; $\mathrm{D}_{3}$-EXE, EXE-19-d3; $17 \beta$-DHE, $17 \beta$-dihydro-EXE; 6 $17 \beta$-DHE-cys, 6 -methylcysteinylandrosta-1,4-diene-17 $\beta$-hydroxy-3-one; $17 \beta$-DHE-Gluc, $17 \beta$-hydroxy-EXE-17-O- $\beta$-D-glucuronide; ER+, estrogen receptor-positive; EXE, exemestane; 6-EXE-cys, 6-methylcysteinylandrosta-1,4-diene-3,17-dione; GSH, L-glutathione; GST, glutathione-Stransferase; $\gamma$-GT, glutamyltranspeptidase; HLC, human liver cytosol; LC-MS, liquid chromatography-mass spectrometry; MS/MS, tandem mass spectrometry; QTOF, Quadrupole Time of Flight; TEM, total quantified EXE metabolite; TLC, thin-layer chromatography; UPLC-MS, ultra-pressure liquid chromatography-mass spectrometry; s, singlet; d, doublet; dd, doublet of doublets; $\mathrm{m}$, multiplet; $\mathrm{J}$, coupling constant; $\delta$, chemical shift. 
highly active in EXE reduction to $17 \beta$-DHE in vitro, and several common variants in the cytosolic keto steroid reductases have been associated with altered enzymatic activity in vitro (Platt et al., 2016; Peterson et al., 2017). Multiple hepatic monooxygenases from cytochrome P450 families 1, 2, and 3 were confirmed to catalyze the production of 6-hydroxymethylandrosta-1,4,6-triene-3,17-dione, 17 $\alpha$-DHE as well as the active metabolite $17 \beta$-DHE (Peterson et al., 2017). CYP4A11 was also found to be responsible for the formation of $17 \beta$-DHE, whereas CYP3A was active in EXE oxidation to form 6-hydroxymethylexemestane (Kamdem et al., 2011).

UGT2B17 is the major enzyme responsible for the glucuronidation of $17 \beta$-DHE, and the $U G T 2 B 17$ deletion polymorphism has been linked to increased levels of $17 \beta$-DHE formation in plasma of women taking EXE (Sun et al., 2010; Luo et al., 2018). Interestingly, whereas drastic decreases in $17 \beta$-hydroxy-EXE-17- $O$ - $\beta$-D-glucuronide (17 $\beta$-DHE-Gluc) levels (e.g., up to 29 -fold in plasma) were associated with increasing numbers of the $U G T 2 B 17$ deletion allele in women taking EXE, only a small (1.3-fold) increase in plasma $17 \beta$-DHE was observed in the same women (Luo et al., 2018). This suggests that other metabolic or excretion pathways may also play a role in EXE metabolism.

The goal of the present study was to characterize other major phase II metabolites of EXE. Results are presented demonstrating the existence of novel phase II cysteine conjugate EXE metabolites in both the urine and plasma of women taking EXE.

\section{Materials and Methods}

Chemicals and Materials. EXE was purchased from Sigma-Aldrich (St. Louis, MO). 17 $\beta$-DHE, 17 $\beta$-DHE-Gluc, $17 \beta$-OH-EXE-d3 (D 3 -17 $\beta$-DHE), $17 \beta$-OH-EXE-d3-17-O- $\beta$-D-glucuronide, and EXE-19-d3 (D -EXE) were purchased from Toronto Research Chemicals (North York, ON, Canada). Ammonium formate was obtained from Sigma-Aldrich, whereas ammonium acetate and formic acid were purchased from Thermo Fisher Scientific (Waltham, MA). Acetonitrile was purchased from Merck (Kenilworth, NJ). Solvent and buffer modifiers for ultra-pressure liquid chromatography-mass spectrometry (UPLC-MS) analysis including acetonitrile, ammonium formate, ammonium acetate, and formic acid were all liquid chromatographymass spectrometry (LC-MS) grade. Milli-Q water (Sigma, St. Louis, MO) was used for the preparation of all solutions. Pooled human liver cytosol (HLC; mixed gender, pool of 50 subjects) was purchased from XENOTECH (Kansas City, KS). Reduced L-glutathione (GSH) and glutamyltranspeptidase $(\gamma$-GT) from equine kidney were purchased from Sigma-Aldrich. L-Cysteine was purchased from Alfa Aesar (Heysham, Lancashire, UK). Preparative C-18 thin-layer chromatography (TLC) plates $(20 \mathrm{~cm} \times 20 \mathrm{~cm} \times 1 \mathrm{~mm})$ were obtained from Sigma-Aldrich. Hexane, diethyl ether, acetonitrile, potassium hydroxide, and methanol used for chemical synthesis were of American Chemical Society reagent grade or higher quality and were purchased from VWR (Radnor, PA).

Subjects and Samples. One hundred and thirty-two postmenopausal breast cancer patients (one Hispanic, two African Americans, two Asians, and 127 Caucasians; age range: $35-89$ years) with ER+ breast tumors were recruited into this study from the Breast Oncology Clinic at the Penn State Hershey Cancer Institute. Approval was obtained from the Institutional Review Board at Penn State University with informed consent obtained from all subjects. Subjects took a single pill ( $25 \mathrm{mg}$ ) of EXE daily (orally) for at least 28 consecutive days and provided blood $(10 \mathrm{ml}$ ) and urine (up to $50 \mathrm{ml}$ ) specimens between 4 and 6 hours after pill ingestion as described previously (Luo et al., 2018). Blood was separated by centrifugation at $1300 \mathrm{~g}$ for 15 minutes at room temperature. Aliquoted plasma and buffy coat fractions of blood samples and aliquoted urine samples were stored at $-80^{\circ} \mathrm{C}$ until analysis. As a control for EXE metabolism, specimens obtained from women not taking EXE who were recruited into other studies at Penn State University College of Medicine (Hershey, PA; $n=10$ ) were also examined to exclude false positives (Ashmore et al., 2013).

Sample Preparation for Identification of EXE Metabolites. Ten urine specimens from subjects taking EXE and 10 urine specimens from control subjects who did not take EXE were selected for analysis. One hundred microliters of $100 \%$ methanol was added to a $50-\mu l$ aliquot of each urine sample to extract EXE and its metabolites. After vortexing and subsequent centrifugation at $16,100 \mathrm{~g}$ for 10 minutes at $4{ }^{\circ} \mathrm{C}$, the supernatant was transferred to a fresh sample vial for analysis by UPLC-MS.

UPLC-MS Conditions for Screening of EXE Metabolites in Urine. For the simultaneous identification of potential novel phase II metabolites and analysis of known metabolites (including EXE, 17 $\beta$-DHE, and 17 $\beta$-DHEGluc), urine samples were prepared as described earlier and analyzed using a UPLC-MS system (Waters, Milford, MA) consisting of an ACQUITY UPLC pump, an ACQUITY FTN (flow through needle) sample manager, an ACQUITY UPLC BEH column C18 $(2.1 \times 100 \mathrm{~mm}, 1.7-\mu \mathrm{m}$ particle size $)$, and a XEVO G2-S Quadrupole Time of Flight (QTOF) mass spectrometer. UPLC was performed at a flow rate of $0.4 \mathrm{ml} / \mathrm{min}$ with solvent A $(5 \mathrm{mM}$ ammonium formate and $0.01 \%$ formic acid in water) and solvent B (100\% acetonitrile) using the following conditions for urine specimens: 0.5 minutes at $25 \%$ solvent B, a linear gradient to $100 \%$ solvent B in 4 minutes, 1.5 minutes at $100 \%$ solvent B followed by re-equilibrium with $25 \%$ solvent $\mathrm{B}$ for 2 minutes. The injection volume of each prepared urine sample was $2 \mu \mathrm{l}$. The column temperature was $35^{\circ} \mathrm{C}$. The Waters XEVO G2-S QTOF MS was equipped with an electrospray ionization probe operated in the positiveion mode, with a capillary voltage at $0.6 \mathrm{kV}$. Nitrogen was used as both the cone and desolvation gases with flow rates maintained at 50 and $800 \mathrm{l} / \mathrm{h}$, respectively. Ultra-pure argon was used as the collision gas with a flow rate of $0.1 \mathrm{l} / \mathrm{h}$. The source and desolvation gas temperatures were $120^{\circ} \mathrm{C}$ and $500^{\circ} \mathrm{C}$, respectively, and the dwell time for each ion was $300 \mathrm{~ms}$. The mass spectrometer was operated in $\mathrm{MS}^{\mathrm{E}}$ mode (MassLynx; Waters) as a nontargeted method for metabolite identification (Plumb et al., 2006). In this method, two interleaved scan functions were used for data acquisition, with both functions collecting data over the same mass range $\left[(\mathrm{m} / \mathrm{z})^{+}=50-1250\right]$. The first scan acquired data using a low collision energy and collected information on the intact (parent) ions in each sample. The second scan acquired data with ramped collision energy from low to high and collected the fragment (daughter) ion data of the ions in the preceding scan (Bateman et al., 2007).

Chemical Synthesis of 6-Methylcysteinylandrosta-1,4-diene-3,17-dione and 6-Methylcysteinylandrosta-1,4-diene-17 $\beta$-hydroxy-3-one. The EXE and $17 \beta$-DHE used for the chemical synthesis of 6-methylcysteinylandrosta-1,4-diene3,17-dione (6-EXE-cys) and 6-methylcysteinylandrosta-1,4-diene-17 $\beta$-hydroxy3-one (6-17 $\beta$-DHE-cys) were synthesized as previously described (Platt et al., 2016). Nuclear magnetic resonance (NMR) spectra were recorded with a Bruker (Billerica, MA) Avance I instrument with $500 \mathrm{MHz}$ for hydrogen and $125 \mathrm{MHz}$ for carbon. The abbreviations for NMR data are the following: s, singlet; d, doublet; $\mathrm{dd}$, doublet of doublets; $\mathrm{m}$, multiplet; $\mathrm{J}$, coupling constant; $\delta$, chemical shift. Chemical shifts were measured based on the residual protium in NMR solvent, and product purity was determined by UPLC spectrum monitored at $254 \mathrm{~nm}$.

1. 6-EXE-cys. EXE $(8.0 \mathrm{mg}, 0.027 \mathrm{mmol})$ and L-cysteine $(9.7 \mathrm{mg}$, $0.080 \mathrm{mmol}$ ) were added to a $10-\mathrm{ml}$ round-bottom flask under the protection of argon, followed by the addition of degassed $1.25 \mathrm{~N}$ potassium hydroxide in $25 \%$ methanol $(1 \mathrm{ml})$. The mixture was stirred at ambient temperature for 24 hours, and the $\mathrm{pH}$ was then adjusted to 5.0 with $2 \mathrm{~N}$ cold $\mathrm{HCl}$. The reaction mixture was subsequently applied on a preparative C-18 reverse-phase TLC plate developing in a chamber containing $20 \%$ acetonitrile in water. The product band determined by 254-nm UV visualization at $R_{\mathrm{f}}=0.3$ was scratched off from the TLC plate with a spatula into a clean $100-\mathrm{ml}$ round-bottom flask. Pure methanol $(40 \mathrm{ml})$ was added to extract the product. After filtration of the methanol-extracted mixture through a fritted funnel and flushing with methanol, the solvent was removed by a rotary evaporator. The residue was washed with $100 \%$ hexane $(2 \times 1 \mathrm{ml})$ and $100 \%$ diethyl ether $(2 \times$ $1 \mathrm{ml})$ and dried to afford the product $(1.5 \mathrm{mg}, \mathrm{Y}=13 \%)$ as an off-white semisolid [ ${ }^{1} \mathrm{H}$ NMR $\left(\mathrm{CD}_{3} \mathrm{OD}\right): \delta 7.31(\mathrm{~d}, \mathrm{~J}=10.2 \mathrm{~Hz}, 1 \mathrm{H}), 6.23\left(\mathrm{dd}, \mathrm{J}_{1}\right.$ $\left.=10.2 \mathrm{~Hz}, \mathrm{~J}_{2}=1.8 \mathrm{~Hz}, 1 \mathrm{H}\right), 6.12(\mathrm{~m}, 1 \mathrm{H}), 3.71(\mathrm{~m}, 1 \mathrm{H}), 3.16-3.19(\mathrm{~m}$, $1 \mathrm{H}), 2.93-3.03(\mathrm{~m}, 2 \mathrm{H}), 2.83(\mathrm{~m}, 1 \mathrm{H}), 2.73(\mathrm{~m}, 1 \mathrm{H}), 2.46(\mathrm{~m}, 1 \mathrm{H})$, $2.35(\mathrm{~m}, 1 \mathrm{H}), 1.95-2.12(\mathrm{~m}, 3 \mathrm{H}), 1.88(\mathrm{~m}, 1 \mathrm{H}), 1.64-1.84(\mathrm{~m}, 3 \mathrm{H})$, $1.25-1.39(\mathrm{~m}, 3 \mathrm{H}), 1.33$ (s, $3 \mathrm{H}), 1.06-1.12(\mathrm{~m}, 1 \mathrm{H}), 0.98(\mathrm{~s}, 3 \mathrm{H}) ;{ }^{13} \mathrm{C}$ DEPT135 (CD $3 \mathrm{OD}): \delta$ 159.6, 127.0, 122.0, 55.0, 51.2, 40.7, $39.9\left(\mathrm{CH}_{2}\right)$, 
36.2 $\left(\mathrm{CH}_{2}\right)$, 35.9, $34.6\left(\mathrm{CH}_{2}\right), 32.1\left(\mathrm{CH}_{2}\right), 23.2\left(\mathrm{CH}_{2}\right), 22.6\left(\mathrm{CH}_{2}\right)$, 18.9, 14.0]. The purity was $>95 \%$.

2. 6-17 $\beta$-DHE-cys. $17 \beta$-DHE $(8.4 \mathrm{mg}, 0.028 \mathrm{mmol})$ and L-cysteine $(9.9 \mathrm{mg}, 0.082 \mathrm{mmol})$ were added to a $10-\mathrm{ml}$ round-bottom flask in a reaction procedure identical to that described earlier for 6-methylcysteinylandrosta-1,4-diene-3,17-dione. The final residue was also washed with hexane $(2 \times 1 \mathrm{ml})$ and diethyl ether $(2 \times 1 \mathrm{ml})$ and dried to afford the product $(1.5 \mathrm{mg}, \mathrm{Y}=13 \%)$ as a white semisolid $\left[{ }^{1} \mathrm{H}\right.$ $\operatorname{NMR}\left(\mathrm{CD}_{3} \mathrm{OD}\right): \delta 7.31(\mathrm{~d}, \mathrm{~J}=10.2 \mathrm{~Hz}, 1 \mathrm{H}), 6.23\left(\mathrm{dd}, \mathrm{J}_{1}=10.2 \mathrm{~Hz}\right.$, $\left.\mathrm{J}_{2}=1.8 \mathrm{~Hz}, 1 \mathrm{H}\right), 6.10(\mathrm{~m}, 1 \mathrm{H}), 3.70(\mathrm{~m}, 1 \mathrm{H}), 3.57\left(\mathrm{dd}, \mathrm{J}_{1}=\mathrm{J}_{2}=8.5 \mathrm{~Hz}\right.$, $1 \mathrm{H}), 3.17(\mathrm{~m}, 1 \mathrm{H}), 2.91-2.99(\mathrm{~m}, 2 \mathrm{H}), 2.80(\mathrm{~m}, 1 \mathrm{H}), 2.70(\mathrm{dd}$, $\left.\mathrm{J}_{1}=12.7 \mathrm{~Hz}, \mathrm{~d}_{2}=6.5 \mathrm{~Hz}, 1 \mathrm{H}\right), 2.22\left(\mathrm{ddd}, \mathrm{J}_{1}=12.4 \mathrm{~Hz}, \mathrm{~J}_{2}=\mathrm{J}_{3}=3.9 \mathrm{~Hz}\right.$, $1 \mathrm{H}), 1.96-1.99(\mathrm{~m}, 1 \mathrm{H}), 1.71-1.90(\mathrm{~m}, 4 \mathrm{H}), 1.68(\mathrm{~m}, 1 \mathrm{H}), 1.63(\mathrm{~m}$, $2 \mathrm{H}), 1.42-1.53$ (m, $2 \mathrm{H}), 1.31$ (s, $3 \mathrm{H}), 0.9-1.2(\mathrm{~m}, 5 \mathrm{H}), 0.83$ (s, $3 \mathrm{H})$; ${ }^{13} \mathrm{C}$ DEPT135 (CD $\left.\mathrm{OD}\right): \delta 160.1,126.8,121.8,81.8,55.4(2 \mathrm{C}), 55.3$, 51.0, $40.9\left(\mathrm{CH}_{2}\right), 40.8,37.4\left(\mathrm{CH}_{2}\right), 36.5,34.7\left(\mathrm{CH}_{2}\right), 30.3\left(\mathrm{CH}_{2}\right), 24.2$ $\left.\left(\mathrm{CH}_{2}\right), 23.6\left(\mathrm{CH}_{2}\right), 18.9,11.4\right]$. The purity was $>95 \%$.

Biosynthesis of 6-EXE-cys, 6-17 $\beta$-DHE-cys, and Deuterium-Labeled Internal Standards. 6-EXE-cys, 6-17 $\beta$-DHE-cys, and their corresponding deuterium-labeled internal standards were biosynthesized in a three-step process involving an initial GSH conjugation reaction of EXE or 17 $\beta$-DHE, removal of glutamic acid from the GSH conjugates using purified active $\gamma$-GT, and subsequent removal of glycine from the cysteinylglycine conjugate using HLC as a source of dipeptidase enzyme.

1. GSH conjugation of EXE and 17 $\beta$-DHE. Pooled HLC (1 mg) was added to a reaction containing $100 \mathrm{mM}$ potassium phosphate $(\mathrm{pH}=7.4)$ and $250 \mu \mathrm{M}$ EXE or $335 \mu \mathrm{M} 17 \beta$-DHE in a total volume of $1 \mathrm{ml}$. The reaction mixture was preincubated at $37^{\circ} \mathrm{C}$ for 3 minutes prior to the addition of $50 \mu \mathrm{l}$ of $100 \mathrm{mM} \mathrm{GSH}$ (final GSH concentration $=5 \mathrm{mM}$ ) (Lash et al., 1999; Zarth et al., 2015; Shi et al., 2016). After 2 hours at $37^{\circ} \mathrm{C}$, the reaction was stopped with an equal reaction volume of ice-cold acetonitrile and centrifuged at $16,100 \mathrm{~g}$ for 10 minutes at $4^{\circ} \mathrm{C}$. Aliquots $(50 \mu 1)$ of the supernatant were injected onto the ACQUITY UPLC BEH C18 column $(2.1 \times 100 \mathrm{~mm}, 1.7-\mu \mathrm{m}$ particle size $)$ for separation by UPLC. The UPLC conditions were the same as those described earlier for screening of EXE metabolites. Conjugate-containing fractions were collected at UPLC retention times of 0.8-2.0 minutes for the EXE-GSH conjugate or $0.5-2.0$ minutes for the $17 \beta$-DHE-GSH conjugate prior to their concentration and removal of organic solvent by speedvac.

2. $\gamma$-GT-mediated removal of $\gamma$-glutamyl from the GSH conjugates of EXE and $17 \beta-D H E$. The EXE-GSH and $17 \beta$-DHE-GSH conjugates collected earlier were digested at $37^{\circ} \mathrm{C}$ for 15 minutes with $1.0 \mathrm{U} / \mathrm{ml} \gamma$-GT, $100 \mathrm{mM}$ potassium phosphate (pH 7.4), $20 \mathrm{mM}$ glycylglycine, and $5 \mathrm{mM}$ dithiothreitol in a total volume of $600 \mu \mathrm{l}$ as previously described (Lash et al., 1999; Del Corso et al., 2006; Grillo et al., 2008). The reaction was stopped with an equal reaction volume of ice-cold acetonitrile and centrifuged at $16,100 \mathrm{~g}$ for 10 minutes at $4^{\circ} \mathrm{C}$, and aliquots $(50 \mu \mathrm{l})$ of the supernatant were injected onto the same UPLC system described earlier. Conjugate-containing fractions were collected at UPLC retention times of 1.0-1.5 minutes for the EXE-cysteinylglycine conjugate and 0.51.0 minute for the $17 \beta$-DHE-cysteinylglycine conjugate prior to their concentration to half their original volume by speedvac.

3. Dipeptidase-mediated removal of glycine from cysteinylglycine conjugates. Collected EXE-cysteinylglycine or $17 \beta$-DHE-cysteinylglycine conjugates were digested with $1.0 \mathrm{mg} / \mathrm{ml}$ pooled HLC in $100 \mathrm{mM}$ potassium phosphate buffer $(\mathrm{pH} 7.4), 0.2 \mathrm{mM} \mathrm{MnCl}_{2}$, and $5 \mathrm{mM}$ dithiothreitol at $37^{\circ} \mathrm{C}$ in a final reaction volume of $200 \mu \mathrm{l}$ (Hirota et al., 1986; Josch et al., 1998; Cappiello et al., 2004; Del Corso et al., 2006). The reaction was stopped with an equal reaction volume of ice-cold acetonitrile and centrifuged at $16,100 \mathrm{~g}$ for 10 minutes at $4^{\circ} \mathrm{C}$, and aliquots $(50 \mu \mathrm{l})$ of the supernatant were injected onto the same UPLC system described earlier.

4. Biosynthesis of $D_{3}-6-E X E$-cys and $D_{3}-6-17 \beta-D H E-c y s$. In enzymatic reactions identical to those described in steps $1-3, \mathrm{D}_{3}$-EXE or $\mathrm{D}_{3}-17 \beta$ DHE was used as the starting material at $330 \mu \mathrm{M}$ in $1-\mathrm{ml}$ reactions. Collected $\mathrm{D}_{3}$-6-EXE-cys and $\mathrm{D}_{3}-6-17 \beta$-DHE-cys conjugates were dried by speedvac and dissolved in pure methanol. The concentrations for the stock solutions of $\mathrm{D}_{3}$-6-EXE-cys and $\mathrm{D}_{3}-6-17 \beta$-DHE-cys, estimated by comparing MS peak area with the chemically synthesized standard, were 2.5 and $10 \mu \mathrm{g} / \mathrm{ml}$, respectively.

Sample Preparation for Quantification of EXE and Its Metabolites in Urine and Plasma. To quantify in vivo levels of EXE and its metabolites, the sample preparation method was modified from that described earlier for metabolite identification. For EXE metabolite analysis in plasma, a 2.5- $\mu$ l aliquot of each plasma sample was first spiked with $2.5 \mu \mathrm{l}$ of a mixture of deuteriumlabeled internal standards in methanol $\left[\mathrm{D}_{3}\right.$-EXE $(0.17 \mu \mathrm{M}), \mathrm{D}_{3}-17 \beta$-DHE $(1.7 \mu \mathrm{M}), 17 \beta$-OH-EXE-d3-17-O- $\beta$-D-glucuronide $(0.52 \mu \mathrm{M}), \mathrm{D}_{3}$-6-EXE-cys $(0.06 \mu \mathrm{M})$, and $\mathrm{D}_{3}-6-17 \beta$-DHE-cys $\left.(0.25 \mu \mathrm{M})\right]$. Twenty microliters of pure methanol was then added to extract EXE and its metabolites and to precipitate proteins. After vortexing and subsequent centrifugation at $16,100 \mathrm{~g}$ for 10 minutes at $4^{\circ} \mathrm{C}, 15 \mu \mathrm{l}$ of the supernatant was transferred to a sample vial and mixed with $15 \mu \mathrm{l}$ of water prior to analysis by UPLC-MS.

For EXE metabolite analysis in urine, a 2.5- $\mu$ l aliquot of each urine sample was first spiked with $2.5 \mu \mathrm{l}$ of the same deuterium-labeled internal standard mixture described earlier. Ten microliters of $75 \%$ methanol was then added to extract EXE and its metabolites. After vortexing and subsequent centrifugation at $16,100 \mathrm{~g}$ for 10 minutes at $4{ }^{\circ} \mathrm{C}, 10 \mu \mathrm{l}$ of the supernatant was transferred to a sample vial and mixed with $5 \mu 1$ of water prior to analysis by UPLC-MS.

UPLC-MS Conditions for Quantification of EXE and Its Metabolites in Urine and Plasma. For the simultaneous quantification of EXE, 17 $\beta$-DHE, 17 $\beta$ DHE-Gluc, 6-EXE-cys, and 6-17 $\beta$-DHE-cys, urine and plasma samples were prepared as described earlier. Using the same UPLC-MS system described earlier, UPLC was performed with solvent A ( $5 \mathrm{mM}$ ammonium formate and $0.01 \%$ formic acid in water) and solvent B (100\% acetonitrile) using the following conditions for both urine and plasma specimens: $0-1.5$ minutes at $25 \%$ solvent $\mathrm{B}$, a linear gradient to $52 \%$ solvent B for 1.5-2.5 minutes, 2.5-4.5 minutes at 52\% solvent B, a linear gradient to $95 \%$ solvent B from 4.5 to 5 minutes, 5-9.5 minutes at $95 \%$ solvent $\mathrm{B}$, followed by a linear gradient to initial conditions of $25 \%$ solvent B from 9.5 to 10 minutes. This was followed by a 1-minute run at $25 \%$ solvent B to equilibrate the column to initial conditions before the next sample injection. The flow rate was $0.4 \mathrm{ml} / \mathrm{min}$ from 0 to 5 minutes, $0.8 \mathrm{ml} / \mathrm{min}$ from 5 to 9.5 minutes, and back to $0.4 \mathrm{ml} / \mathrm{min}$ from 9.5 to 10 minutes. The injection volume of each prepared urine and plasma sample was $5 \mu$ l. The column temperature was $35^{\circ} \mathrm{C}$. The Waters XEVO G2-S QTOF MS was operated in tandem mass spectrometry (MS/MS) mode, with the electrospray ionization probe operated in the positiveion mode and a capillary voltage at $0.6 \mathrm{kV}$. The cone and desolvation gas flow rates were maintained at 50 and $800 \mathrm{l} / \mathrm{h}$, respectively, and the collision gas flow rate was $0.1 \mathrm{l} / \mathrm{h}$. The source and desolvation gas temperatures were $120^{\circ} \mathrm{C}$ and $500^{\circ} \mathrm{C}$, respectively, and the dwell time for each ion was $300 \mathrm{~ms}$. The ion-related parameters for the 10 transitions monitored are listed in Table 1 .

The limits of quantification for EXE, 17 $\beta$-DHE, 17 $\beta$-DHE-Gluc, 6-EXE-cys, and 6-17 $\beta$-DHE-cys were $2.1,1.6,1.2,0.7$, and $7.2 \mathrm{nM}$ in plasma, respectively, and $2.1,1.6,6.3,1.5$, and $7.2 \mathrm{nM}$ in urine, respectively. Standard curves were constructed by plotting the ratio of analyte peak area to peak area of the corresponding internal standard (described earlier) versus concentration of analyte standard. The concentrations of stock standards were $1000 \mathrm{ppm}$. A serial dilution of standards at concentrations ranging from $1.6 \mathrm{nM}$ to $1.7 \mu \mathrm{M}, 1.6 \mathrm{nM}$ to $1.7 \mu \mathrm{M}$,

\section{TABLE 1}

MS/MS transitions and ion optic parameters for EXE and EXE metabolites

\begin{tabular}{lccc}
\hline & ES+ MS/MS Transition & Cone Voltage & Collision Energy \\
\hline & $(m / z)+$ & $V$ & $e V$ \\
EXE & $297.19>297.19$ & 25 & 10 \\
D $_{3}$-EXE & $300.20>300.20$ & 25 & 10 \\
17 $\beta$-DHE & $299.20>299.20$ & 25 & 10 \\
D $_{3}$-17 $\beta$-DHE & $302.22>302.22$ & 25 & 10 \\
17 $\beta$-DHE-Gluc & $475.23>281.19$ & 20 & 15 \\
D $_{3}$-17 $\beta$-DHE-Gluc & $478.25>284.21$ & 20 & 15 \\
6-EXE-cys & $418.21>297.19$ & 15 & 15 \\
D $_{3}$-6-EXE-cys & $421.22>300.20$ & 15 & 15 \\
6-17 $\beta$-DHE-cys & $420.22>299.20$ & 15 & 15 \\
D $_{3}$-6-17 $\beta$-DHE-cys & $423.24>302.22$ & 15 & 15 \\
\hline
\end{tabular}

$\mathrm{D}_{3}$-17 $\beta$-DHE-Gluc, $17 \beta$-OH-EXE-d3-17-O- $\beta$-D-glucuronide. 
$1.0 \mathrm{nM}$ to $2.1 \mu \mathrm{M}, 0.7 \mathrm{nM}$ to $12 \mu \mathrm{M}$, and $4.6 \mathrm{nM}$ to $2.4 \mu \mathrm{M}$ was used to establish standard curves for EXE, 17 $\beta$-DHE, 17 $\beta$-DHE-Gluc, 6-EXE-cys, and 6-17 $\beta$-DHEcys, respectively. Analyte concentrations were determined by measuring the peak area ratios of analyte to internal standard and then calculating analyte concentration from the appropriate standard curve using the Waters TargetLynx software. Urinary creatinine was measured as previously described (Luo et al., 2018).

Statistical Analysis. Calculations of mean and S.E. were performed using Prism (version 7; GraphPad Software, San Diego, CA).

\section{Results}

Identification of EXE and 17 $\beta$-DHE Conjugates. Urine specimens from subjects taking EXE were extracted and analyzed by UPLC-MS using $\mathrm{MS}^{\mathrm{E}}$, a nontargeted method for metabolite identification that allows for the scanning of both intact ions (channel 1) and fragment ions (channel 2) simultaneously over the same mass range.

As shown by representative chromatographs in Fig. 1, the $\mathrm{MS}^{\mathrm{E}}$ method applied in this study was sensitive enough to detect known EXE metabolites extracted from the intact ion channel 1 including EXE (Fig. $1 \mathrm{~A}$; extracted mass $=297.19), 17 \beta-\mathrm{DHE}($ Fig. $1 \mathrm{C}$; extracted mass = 299.20), and 17 $\beta$-DHE-Gluc (Fig. 1E; extracted mass $=475.23$ ) in the urine from subjects taking EXE. All peaks corresponded to those observed for EXE, 17 $\beta$-DHE, and 17 $\beta$-DHE-Gluc standards (Fig. 1, B, $\mathrm{D}$, and $\mathrm{F}$, respectively); none were observed in the urine of subjects not taking EXE (results not shown).

In screening for novel EXE conjugates, the MS trace of $(\mathrm{m} / \mathrm{z})^{+}$ $=297.185$ for $[\mathrm{EXE}+\mathrm{H}]^{+}$, the common fragment ion for EXE conjugates, was extracted from fragment ion channel 2 (Fig. 2A). In addition to the expected peak for EXE (retention time 3.20 minutes), two major peaks (termed peaks 1 and 2, retention times $=1.33$ and 1.74 minutes, respectively) were observed. Neither of these peaks were detected in urine specimens from control subjects not taking EXE (results not shown). Molecular ions for compounds that correspond to these two fragment peaks were then searched within the intact ion channel (channel 1); the retention times of peaks 1 and 2 in the intact (parent) ion channel 1 (Fig. 2B) matched that observed for peaks 1 and 2 in fragment ion channel 2 (Fig. 2A). The corresponding mass spectrum of the parent ion for peak 1 from the intact (parent) ion channel 1 showed a major peak with an $(\mathrm{m} / \mathrm{z})^{+}=418.2060$, presumably the $[\mathrm{X}+\mathrm{H}]^{+}$ion, where $\mathrm{X}$ refers to an unknown EXE conjugate (Fig. 2C). Two additional accurate mass peaks were observed (Fig. 2C), with $(\mathrm{m} / \mathrm{z})^{+}=440.1874$ and 456.1615, likely corresponding to the $(\mathrm{m} / \mathrm{z})^{+}$for $[\mathrm{X}+\mathrm{Na}]^{+}$and $[\mathrm{X}+\mathrm{K}]^{+}$, respectively. The accurate mass spectrum for peak 2 (data not shown) was identical to that observed for peak 1 (Fig. 2C), suggesting that the two peaks are isomers of the same EXE conjugate. The trace of 418.206 extracted from the intact (parent) ion scan from channel 1 (from Fig. 2C) exhibited two peaks (Fig. 2D) that matched the retention times of peaks 1 and 2 extracted from fragment ion channel 2 for $(\mathrm{m} / \mathrm{z})^{+}=297.185$ (Fig. 2A). Peak 1 was observed in all 10 urine specimens screened by $\mathrm{MS}^{\mathrm{E}}$, whereas peak 2 was detected in seven of the 10 urine specimens.

The potential composition for the parent ion of $(\mathrm{m} / \mathrm{z})^{+}=418.2057$ was calculated using the Elemental Composition tool within MassLynx 4.1. Since the composition of EXE is $\mathrm{C}_{20} \mathrm{H}_{24} \mathrm{O}_{2}$, the molecular composition of possible EXE conjugates had to contain $\geq 20$ carbons and $\geq 2$ oxygens. The only candidate composition available that matched these criteria was $\mathrm{C}_{23} \mathrm{H}_{32} \mathrm{NO}_{4} \mathrm{~S}\left[(\mathrm{~m} / \mathrm{z})^{+}=418.2052\right]$, which exactly matched the predicted structure corresponding to the cysteine conjugate of EXE. Therefore, compounds responsible for peaks 1 and 2 in the urine of women taking EXE were predicted to be EXE-cys isomers $\left(\mathrm{C}_{23} \mathrm{H}_{31} \mathrm{NO}_{4} \mathrm{~S}\right)$.

For identifying conjugates of $17 \beta$-DHE, an approach similar to that used for identifying EXE conjugates was undertaken. A trace corresponding to $(\mathrm{m} / \mathrm{z})^{+}=299.201$ for $[17 \beta-\mathrm{DHE}+\mathrm{H}]^{+}$, the common fragment ion for $17 \beta$-DHE conjugates, was extracted from fragment ion channel 2 (Fig. 3A), whereas a trace corresponding to the intact (parent) ion for $17 \beta$-DHE-cys $\left[(\mathrm{m} / \mathrm{z})^{+}=420.221\right]$ was extracted from the intact (parent) ion channel 1 (Fig. 3B). Two fragment ion peaks were observed in the channel 2 trace (peak 3 at 0.86 minutes and peak 4 at 1.23 minutes; Fig. 3A) that aligned exactly with the retention times of peaks extracted from the intact (parent) ion $\left[(\mathrm{m} / \mathrm{z})^{+}=420.2209\right]$ trace in channel 1 (Fig. 3B). The mass spectrum extracted for peak 3 from the intact (parent) ion channel 1 is shown in Fig. 3C. The peaks of $(\mathrm{m} / \mathrm{z})^{+}=$ $420.2209,442.2027$, and 458.1770 likely corresponded to the ions of $[17 \beta \text {-DHE-cys }+\mathrm{H}]^{+},[17 \beta \text {-DHE-cys }+\mathrm{Na}]^{+}$, and $[17 \beta \text {-DHE-cys }+\mathrm{K}]^{+}$, respectively. Similar to that observed for EXE-cys conjugates, peak 3 was observed in all 10 urine samples screened by the $\mathrm{MS}^{\mathrm{E}}$, whereas peak 4 was observed in seven of the 10 urine samples. Again, neither of these peaks were detected in urine samples from control subjects not taking EXE (results not shown). Therefore, compounds responsible for peaks 3 and 4 were predicted to be $17 \beta$-DHE-cys isomers $\left(\mathrm{C}_{23} \mathrm{H}_{33} \mathrm{NO}_{4} \mathrm{~S}\right)$.

Chemical Synthesis of Cysteine Conjugates of EXE and 17 $\boldsymbol{\beta}$-DHE. To confirm the structures of the predicted cysteine conjugates of EXE and 17 $\beta$-DHE identified earlier, 6-EXE-cys and $6-17 \beta$-DHE-cys were synthesized chemically. As described in the Materials and Methods, the structures of both chemically synthesized cysteine conjugates were confirmed by NMR, and the purity of both conjugates was confirmed by LC-MS to be $>95 \%$ (results not shown).

The retention times for peaks corresponding to chemically synthesized 6-EXE-cys and 6-17 $\beta$-DHE-cys (Fig. 4, A and B, respectively) matched those observed for the predicted EXE-cys and $17 \beta$-DHE-cys peaks 1 and 3, respectively (Figs. 2D and 3B, respectively), detected in the urine of subjects taking EXE. In addition, coelution experiments were performed by adding chemically synthesized 6-EXE-cys and 6-17 $\beta$-DHE-cys into urine specimens of EXE-treated subjects. LC-MS analysis showed increases in peak size for each compound (results not shown), further demonstrating that the urinary peaks are identical to the chemically synthesized standards. Together, these data suggest that the MS peaks 1 and 3 (Figs. 2D and 3B, respectively) from urine of women taking EXE corresponded to 6-EXE-cys and 6-17 $\beta$-DHE-cys.

Biosynthesis of Cysteine Conjugates of EXE and 17 $\beta$-DHE. GSH is a tripeptide ( $\gamma$-glu-cys-gly) that can conjugate to an electrophilic substrate, which can be further metabolized to form cysteine conjugates during mercapturic acid biosynthesis (Hinchman and Ballatori, 1994; van Bladeren, 2000; Hayes et al., 2005). To explore whether this mechanism of cysteine conjugate formation may occur for EXE and 17 $\beta$-DHE in vivo, cysteine conjugates of EXE and $17 \beta$-DHE were enzymatically synthesized in a three-step reaction. For the synthesis of EXE-cys, the EXE-glutathione conjugate was first synthesized by incubating EXE with GSH in the presence of HLC as a source of glutathione-S-transferases (GSTs). Products of the reaction were analyzed by LC-MS. Three peaks (retention times $=1.15,1.60$, and 1.81 minutes) were detected as potential EXEglutathione conjugates $\left[(\mathrm{m} / \mathrm{z})^{+}=604.27\right.$; results not shown]. Using LC-purified peak 1 (retention time $=1.15$ minutes), EXE-cys conjugates were then formed (Fig. 4C) after two additional reactions using purified $\gamma$-GT in reaction 1 and then HLC for reaction 2. The retention time observed for the enzymatically synthesized EXE-cys conjugate (1.31 minutes) was similar to that observed for the chemically synthesized 6-EXE-cys conjugate (1.33 minutes; Fig. 4A) and was similar to that observed for the putative 6-EXE-cys conjugate corresponding to peak 1 in the urine of subjects taking EXE (Fig. 2D).

Using $17 \beta$-DHE as a substrate, a similar three-step reaction approach was performed to enzymatically synthesize the 6-17 $\beta$ DHE-cys conjugate. The retention time observed for the enzymatically synthesized 6-17 $\beta$-DHE-cys conjugate (0.84 minutes; Fig. 4D) 
A

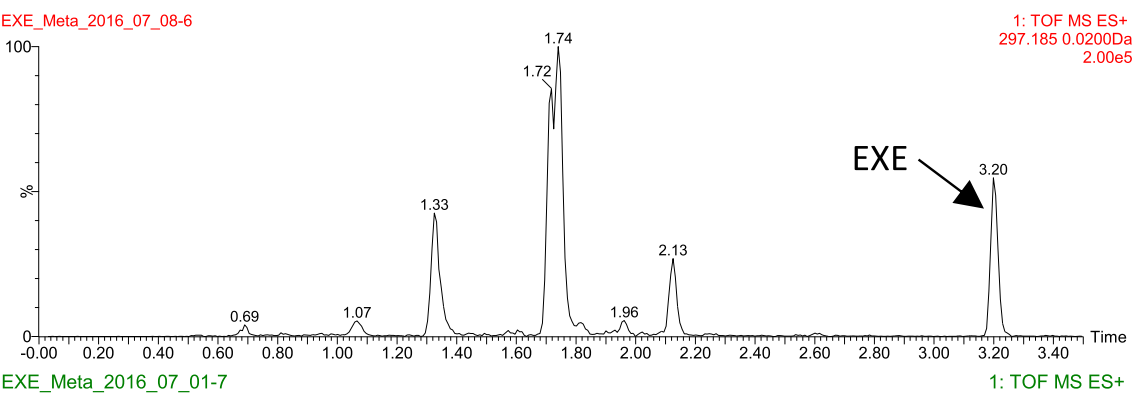

B XE_Meta_2016_07_01-7

B ${ }^{100}$

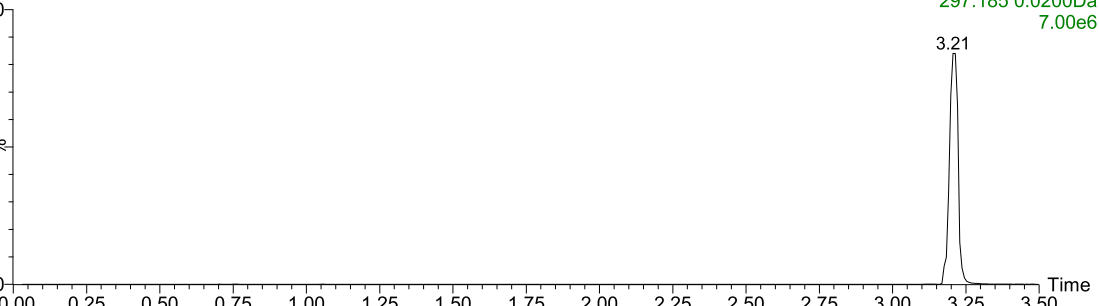
EXE_Meta_2016_07_08-6

C

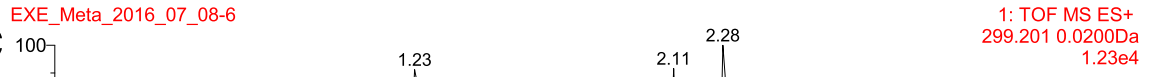

\section{D}

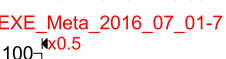

EXE_Meta_2016_07_01-7

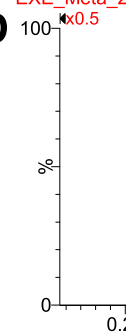

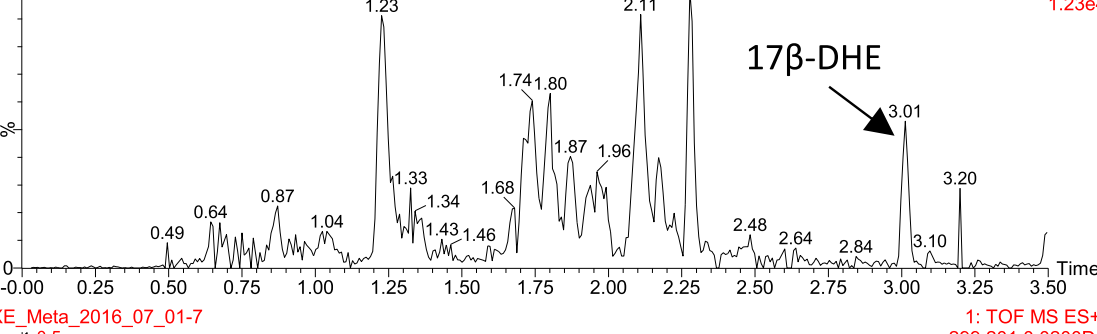

$299.2010 .0200 D^{2}$

Fig. 1. UPLC/MS ${ }^{\mathrm{E}}$ analysis of EXE metabolites in urine from a subject taking $\operatorname{EXE}(\mathrm{A}, \mathrm{C}$, and $\mathrm{E})$ versus chemical standards (B, D, and F). (A and B) Extracted ion chromatogram of 297.185 (EXE) from channel 1 (intact ion). (C and D) Extracted ion chromatogram of 299.201 (17 $\beta$-DHE) from channel 1. (E and $\mathrm{F})$ Extracted ion chromatogram of 475.23 (17 $\beta$-DHEGluc) from channel 1. TOF, Time of Flight.

Meta_2016 07 08-6

E

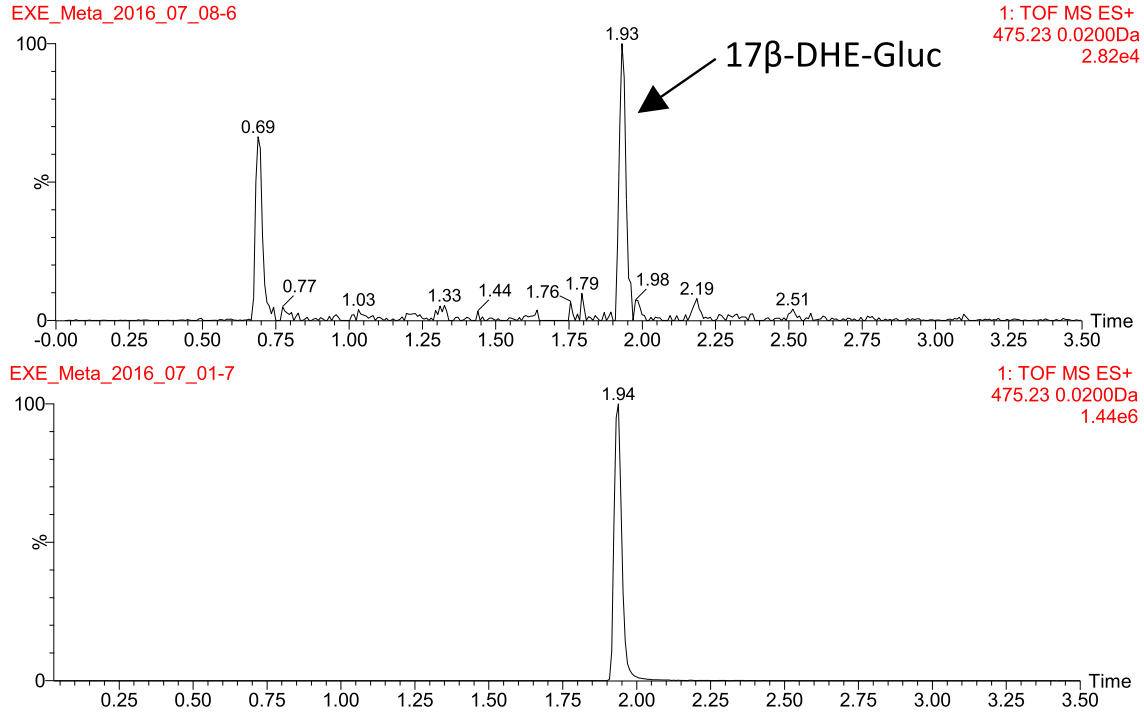

was similar to that observed for the chemically synthesized 6-17 $\beta$ DHE-cys conjugate (Fig. 4B) and to that observed for the putative $17 \beta$-DHE-cys conjugate corresponding to peak 3 in the urine of subjects taking EXE (Fig. 3B). In addition, a similar pattern was observed for both the enzymatically synthesized $\mathrm{D}_{3}$-labeled 6-EXEcys and 6-17 $\beta$-DHE-cys conjugates (Fig. 4, E and F, respectively). All cysteine conjugates were confirmed by analysis of corresponding mass spectra (results not shown). 


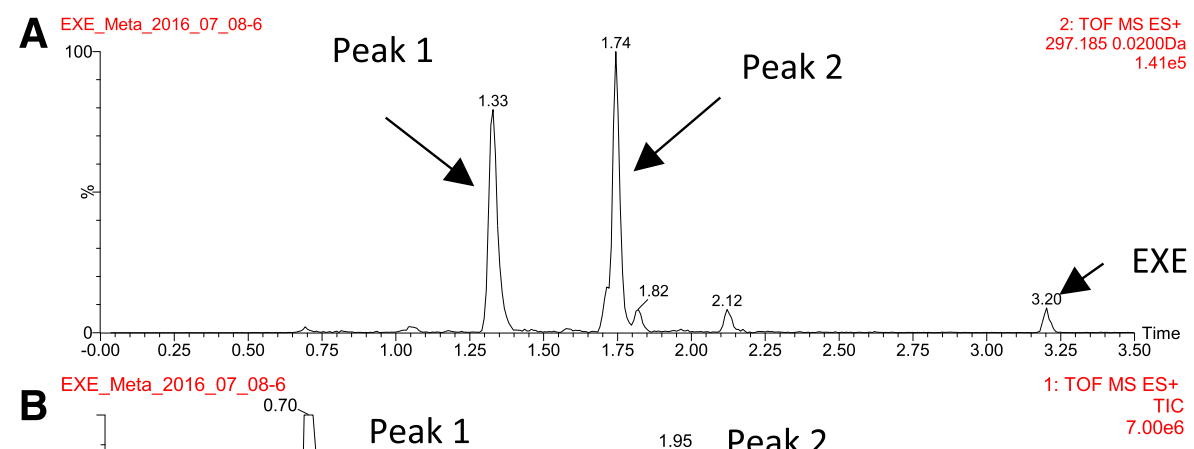

B
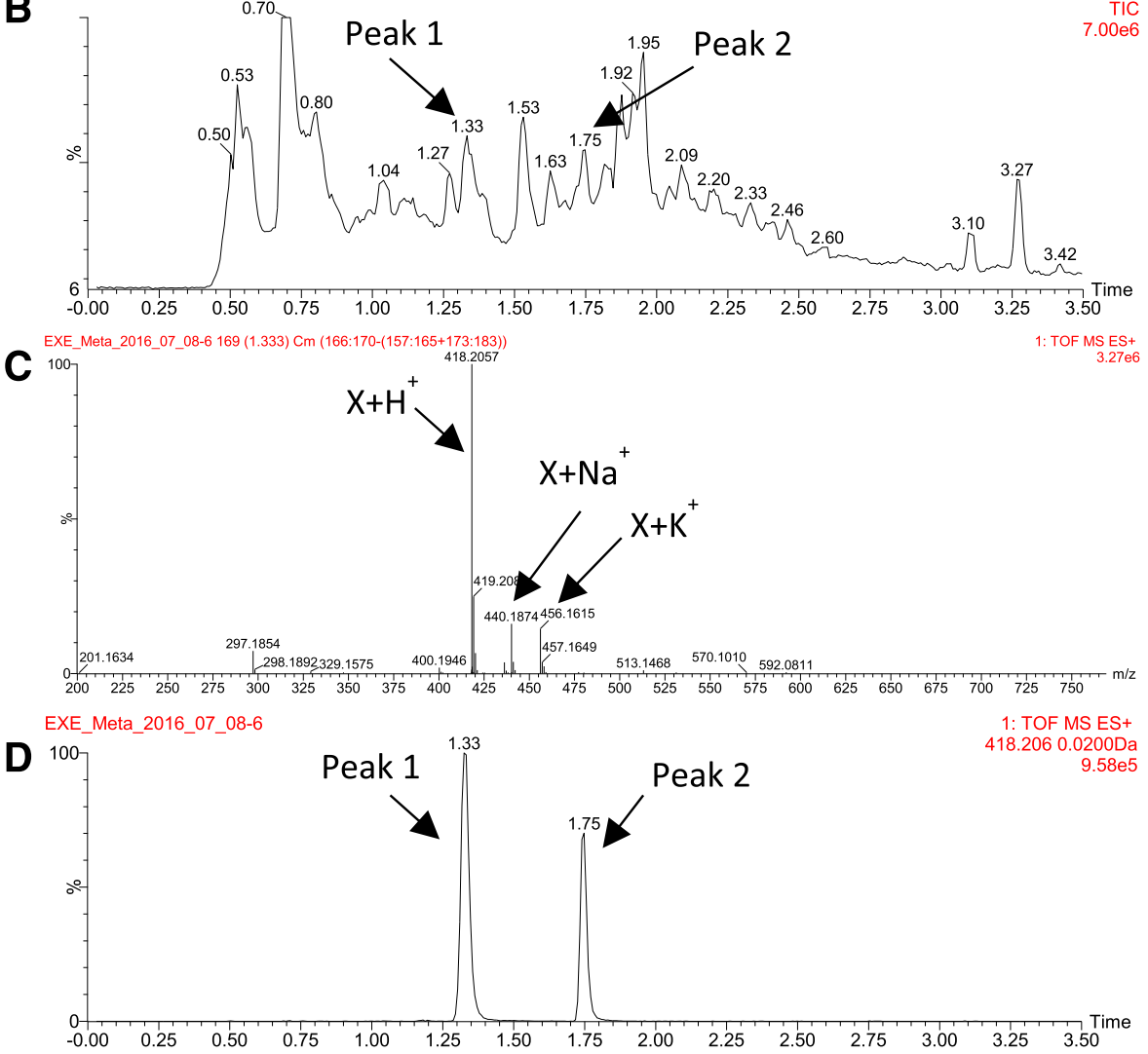

Fig. 2. UPLC/MS ${ }^{\mathrm{E}}$ analysis for identification of $\mathrm{EXE}$ conjugates in urine from a subject taking EXE. (A) Extracted ion chromatogram of 297.185 from channel 2 (fragment ions in screening for EXE conjugates). (B) Chromatogram of total intact ions of channel 1. (C) Mass spectrum for peak 1 in (B). (D) Extracted ion chromatogram of 418.206 from channel 1. TIC, Total Ion Current; TOF, Time of Flight.

Quantification of EXE and Its Metabolites In Vivo. For quantification of EXE and its metabolites in vivo, EXE metabolites were analyzed by UPLC-MS/MS for all urine samples and matched plasma samples from 132 postmenopausal breast cancer patients who had taken $25 \mathrm{mg}$ of EXE per day for at least 4 weeks. As shown for representative MS chromatograms of EXE metabolites from a subject taking EXE (Fig. 5), the retention times were 4.02 minutes for EXE, 3.73 minutes for $17 \beta$-DHE, 2.65 minutes for $17 \beta$-DHE-Gluc, 1.34 minutes for 6-EXE-cys, and 0.88 minutes for 6-17 $\beta$-DHE-cys for both urine and plasma using this UPLC-MS/MS method. In all cases, the retention times of each peak were similar to that of their corresponding internal standard peaks (see Figs. 1 and 4). Although the predicted isomers of EXE-cys and 17 $\beta$-DHE-cys (peaks 2 and 4 in Figs. 2D and 3B, respectively) were not quantified due to a lack of accurate standards, they may be present at similar or higher levels than that observed for 6-EXE-cys and 6-17 $\beta$-DHE-cys if their MS response factors are similar to 6-EXE-cys and 6-17 $\beta$-DHE-cys, respectively.

The major metabolites in the plasma of subjects treated with EXE were $17 \beta$-DHE-Gluc (mean $=30 \mathrm{nM}$; Table 2 ) and 6-EXE-cys $($ mean $=22 \mathrm{nM})$. The levels of 6-17 $\beta$-DHE-cys $(5.9 \mathrm{nM})$ and $17 \beta$ DHE $(2.5 \mathrm{nM})$ were lower than that observed for the parent EXE
$($ mean $=14 \mathrm{nM})$ in plasma, with the average percentage of $17 \beta$-DHE in total quantified EXE metabolites (TEM) observed at 3-fold lower levels than that observed for $17 \beta$-DHE-Gluc. The mean levels of the combined cysteine conjugates for plasma EXE plus 17 $\beta$-DHE were roughly equivalent to the levels of plasma $17 \beta$-DHE-Gluc, with the average percentage of TEM ranging from $35 \%$ to $36 \%$ for both (6-EXE-cys $+6-17 \beta$-DHE-cys) and $17 \beta$-DHE-Gluc. The percentages of $17 \beta$-DHE-Gluc and 6-EXE-cys in TEM (36\% and $23 \%$, respectively) were highest when compared with other EXE metabolites in the plasma of EXE-treated subjects, ranging from $12 \%$ to $17 \%$ for $17 \beta$-DHE, $6-17 \beta$-DHE-cys, and EXE.

Although a similar trend was observed for urinary EXE metabolites, the levels of urinary $17 \beta$-DHE-Gluc were 5.5 -fold less than the combined cysteine conjugates for urinary EXE plus $17 \beta$-DHE, which were the major urinary metabolites in women taking EXE (Table 2). $17 \beta$-DHE-Gluc comprised, on average, $21 \%$ of total quantified urinary EXE metabolites versus the combined 6-EXE-cys plus 6-17 $\beta$-DHEcys conjugates, which comprised $77 \%$ of total quantified urinary EXE metabolites. The levels of urinary $17 \beta$-DHE-Gluc $(1.4 \mathrm{nmol} / \mathrm{mg}$ creatinine) were slightly lower than those observed for 6-17 $\beta$-DHE-cys $(1.8 \mathrm{nmol} / \mathrm{mg}$ creatinine), which was 3.3 -fold lower than that observed 
A

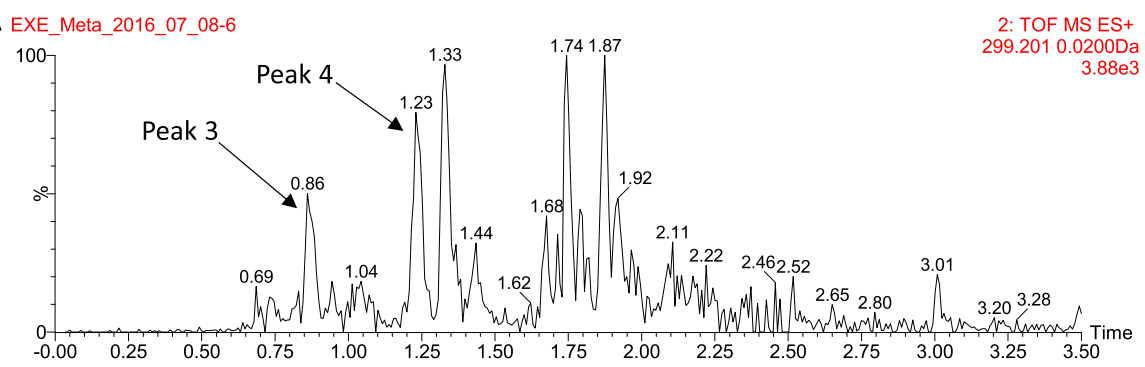

B

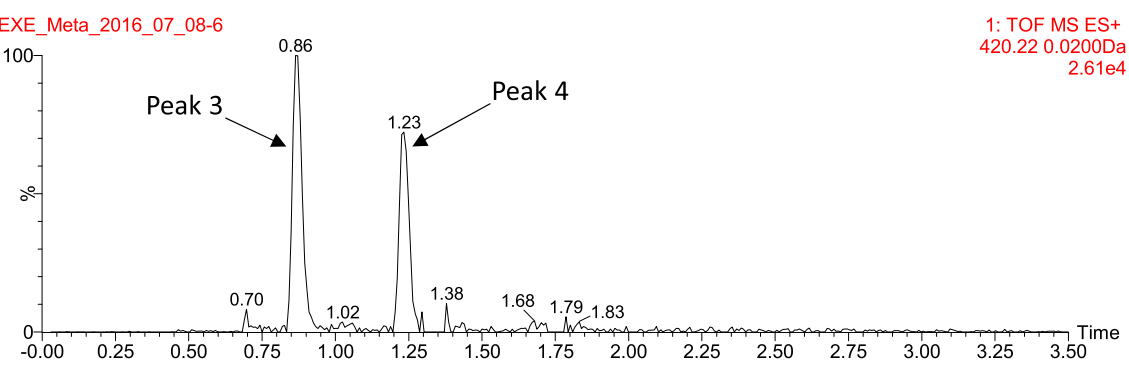

C

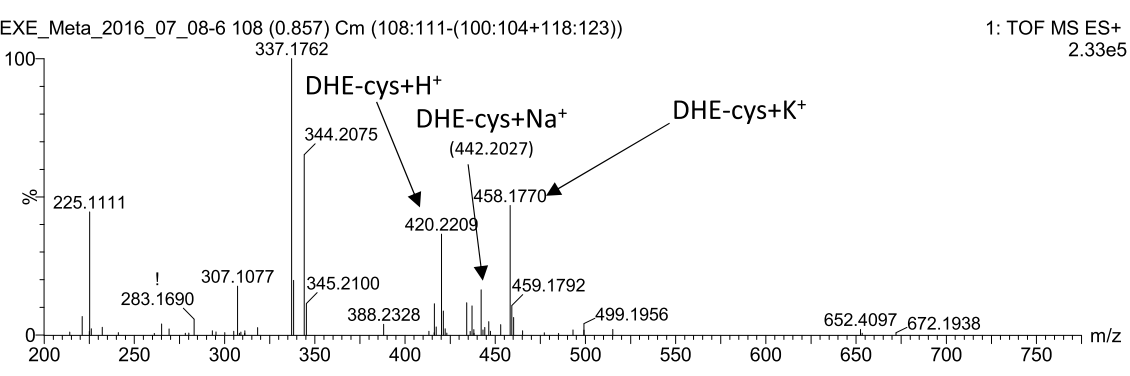

Fig. 3. UPLC/MS ${ }^{\mathrm{E}}$ analysis for identification of $17 \beta$ DHE conjugates from a subject taking EXE. (A) Extracted ion chromatogram of 299.201 from channel 2 (fragment ions in screening for $17 \beta$-DHE conjugates). (B) Extracted ion chromatogram of 420.22 from channel 1. (C) Mass spectrum for peak 3 in (B). TOF, Time Of Flight. for the major urinary metabolite 6-EXE-cys. The mean levels of urinary 6-EXE-cys $($ mean $=5.9 \mathrm{nmol} / \mathrm{mg}$ creatinine) were almost 900 -fold higher than the urinary metabolite observed at the lowest level, $17 \beta$ DHE (mean $=0.0066 \mathrm{nmol} / \mathrm{mg}$ creatinine) .

\section{Discussion}

Previous studies indicated that the major mode of metabolism of EXE is by reduction to form $17 \beta$-DHE and UGT2B17-mediated glucuronidation to form 17 $\beta$-DHE-Gluc (Sun et al., 2010; Luo et al., 2018). In the present study, two novel major EXE metabolites were identified-the cysteine conjugates of EXE (6-EXE-cys) and 17 $\beta$-DHE (6-17 $\beta$-DHEcys). The mean levels of the combined 6-EXE-cys plus 6-17 $\beta$-DHE-cys were 5.5-fold higher than those observed for $17 \beta$-DHE-Gluc in urine and were similar to the levels of $17 \beta$-DHE-Gluc observed in plasma. 6-EXE-cys formed the major cysteine conjugate of EXE, comprising $77 \%$ and $79 \%$ of the total mean cysteine conjugate levels in urine and plasma, respectively. This suggests that the formation of cysteine conjugates is the major excretion pathway for EXE in humans, with 6-EXE-cys the major urinary EXE metabolite (see Scheme 1).

Although the stereochemistry at the 6-position was not assigned in the current paper, we speculate the stereo orientation for the 6 substitute is " $\alpha$ " for both synthesized 6-EXE-cys and 6-17 $\beta$-DHE-cys based on the proton coupling constant between the proton at the 4-position and the proton at the 6-position of both EXE-cys and 17 $\beta$-DHE-cys conjugates. For 6-substituted steroids, long-distance coupling between H-4 and H-6 in ${ }^{1} \mathrm{H}$ NMR will be observed only when the 6-substituent is in the $\alpha$ position (Chin and Warren, 1972; Schneider et al., 1973; Numazawa and Oshibe, 1994; Numazawa and Yamaguchi, 1998; Görlitzer et al., 2006). In an analysis of a series of 6-substituted phenylaliphatic steroids,
Numazawa and Yamaguchi (1998) demonstrated that proton coupling between the hydrogens at the 4- and 6-positions was only observed for the $6 \alpha$-substituted, but not the $6 \beta$-substituted, phenylaliphatic steroids. Similar C-4 proton signals have been reported for a series of 6-alkyl-, 6-bromo-, and 6-(bromoacetoxy)androstenediones (Numazawa and Oshibe, 1994; Numazawa and Yamaguchi, 1998). In addition, the EXE derivative $6 \alpha$-chlormethylandrosta-1,4-dien-3,17-dion exhibits similar long-distance coupling between $\mathrm{H}-4$ and $\mathrm{H}-6$ (Görlitzer et al., 2006). These data are consistent with the proton coupling pattern of 6-EXE-cys and 6-17 $\beta$-DHE-cys observed by ${ }^{1} \mathrm{H}$ NMR in the present study (results not shown), suggesting that both conjugates were in the $\alpha$-position. However, X-ray crystallography analysis of the two conjugates will provide a more definitive validation of their structure.

Previous in vitro studies in a panel of human liver microsomes demonstrated that deletion of the UGT2B17 gene resulted in significant decreases in 17 $\beta$-DHE-Gluc formation (Sun et al., 2010). Although drastic decreases in urinary and plasma $17 \beta$-DHE-Gluc levels were also associated with increasing numbers of the $U G T 2 B 17$ deletion allele in women taking EXE (e.g., up to 29-fold in plasma), only a small (1.3-fold) corresponding increase in plasma $17 \beta$-DHE was observed in the same women (Luo et al., 2018). In the present study, 17 $\beta$-DHE-Gluc was demonstrated to comprise $36 \%$ of the total quantified EXE metabolites in plasma, levels that were approximately equal to that observed for both of the cysteine conjugates combined (which comprised $35 \%$ of the total quantified plasma EXE metabolites) and only slightly more than that observed for 6-EXE-cys alone (which comprised 23\% of total quantified plasma EXE metabolites). In addition, $17 \beta$-DHE-Gluc comprised only $21 \%$ of the total quantified EXE metabolites in urine. These values correspond with the fact that either no alterations or only small increases 


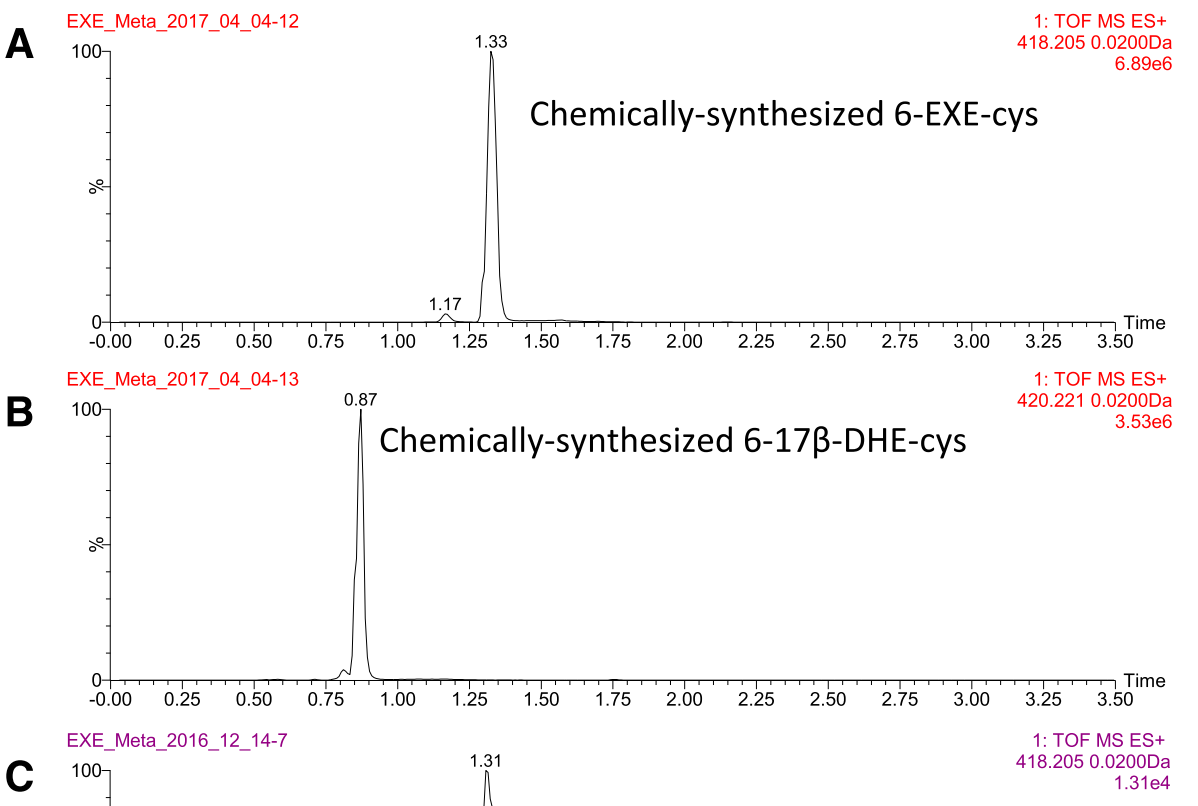

Biosynthesized EXE-cys

D

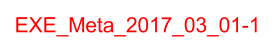

EXE_Meta_2017_03 01-1

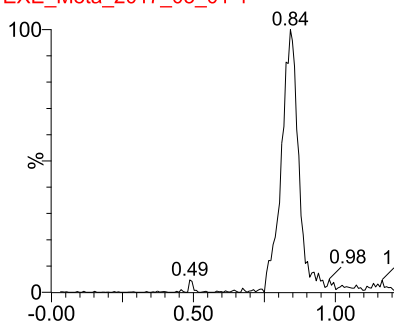

E

EXE_Meta_2017_03_24-1

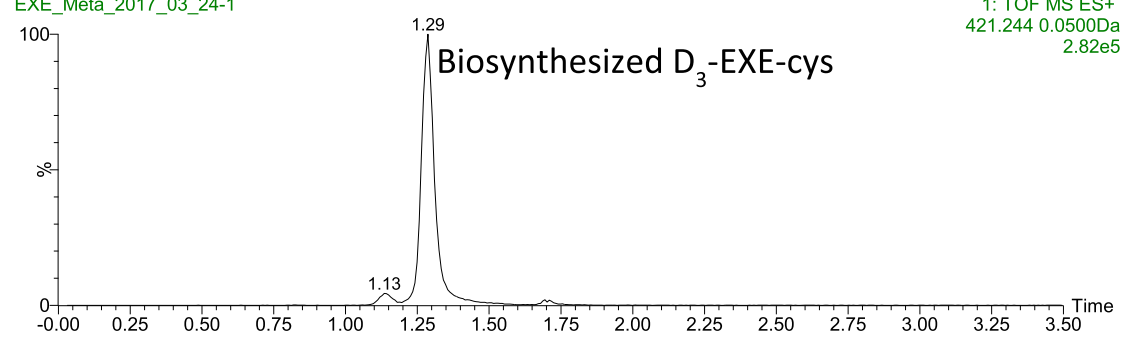

F

EXE_Meta_2017_03_06-8

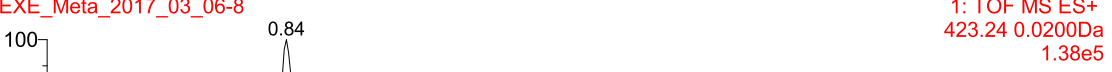

Biosynthesized $D_{3}-17 \beta-D H E-c y s$
Biosynthesized $17 \beta$-DHE-cys

1: TOF MS ES+ 420.221 0.0200Da $2.01 \mathrm{e} 4$

\section{7}
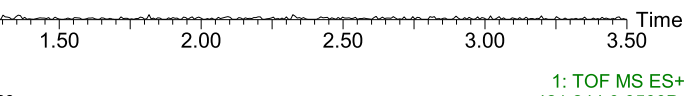

D00Da

$82 \mathrm{e} 5$

Fig. 4. UPLC/MS ${ }^{\mathrm{E}}$ analysis of chemically synthesized and biosynthesized EXE and $17 \beta$-DHE cysteine conjugates. (A) MS (418.205) chromatography for chemically synthesized 6-EXE-cys standard. (B) MS (420.221) chromatography for chemically synthesized 6-17 $\beta$-DHEcys standard. (C) MS (418.205) chromatography for biosynthesized EXE-cys. (D) MS (420.221) chromatography for biosynthesized $17 \beta$-DHE-cys. (E) MS (421.244) chromatography for biosynthesized $\mathrm{D}_{3}$-EXE-cys. (F) MS (423.240) chromatography for biosynthesized $D_{3}-17 \beta$ DHE-cys.

in urinary or plasma EXE and $17 \beta$-DHE were observed in subjects taking EXE who were homozygous for the UGT2B17 deletion polymorphism (i.e., with no active UGT2B17) in previous studies (Luo et al., 2018).
A three-step metabolism pathway similar to the first three steps of the mercapturic acid synthesis pathway was shown to be a viable in vivo mechanism of EXE and 17 $\beta$-DHE cysteine conjugate formation in the 


\section{A. Urinary Metabolites}
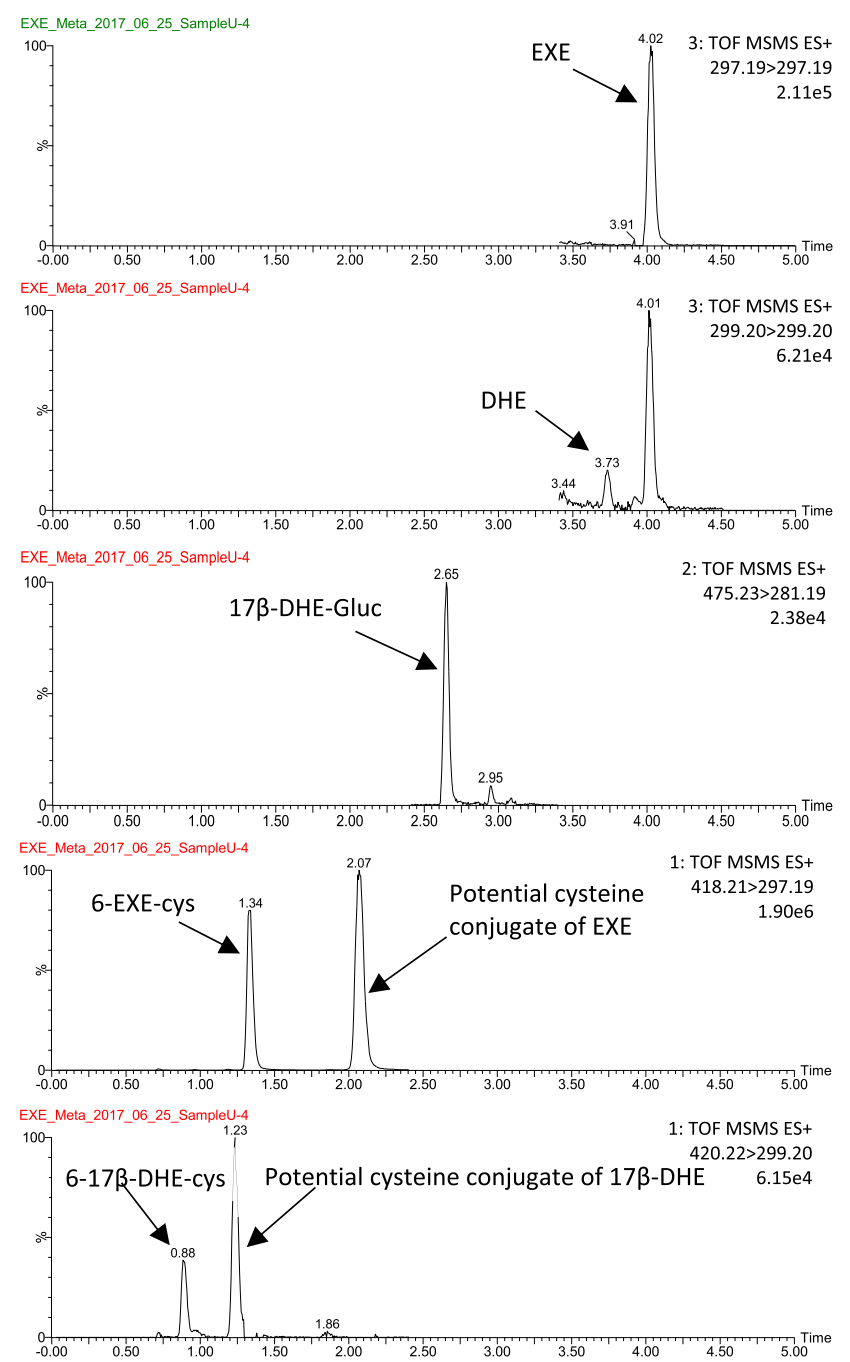

\section{B. Plasma Metabolites}
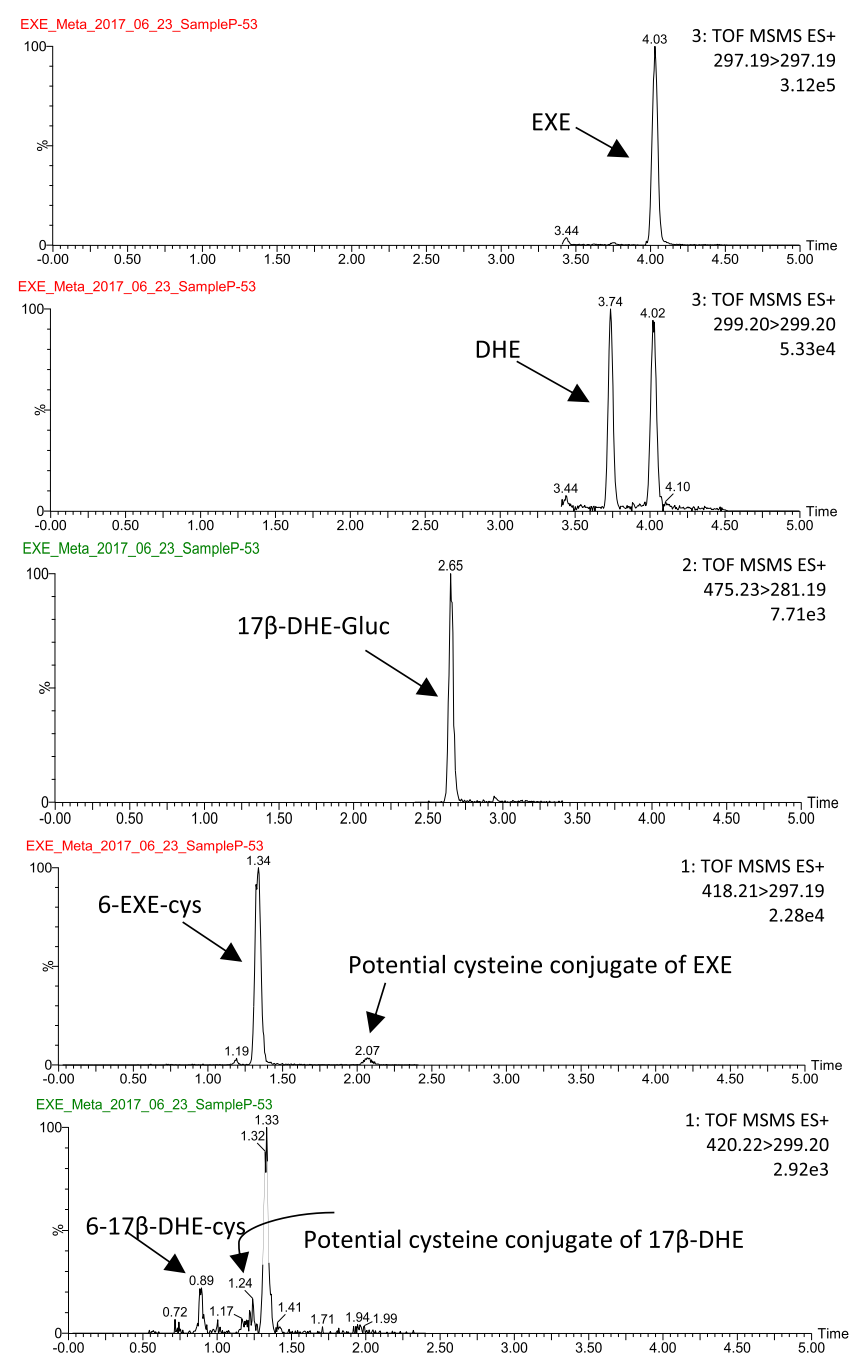

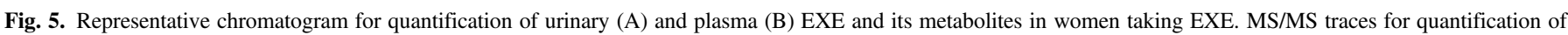

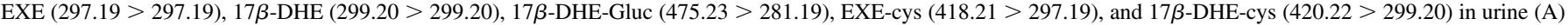
and in plasma (B) are shown.

present studies. The three steps include an initial GSH conjugation reaction catalyzed by GSTs to form EXE-GSH or $17 \beta$-DHE-GSH, a second reaction catalyzed by $\gamma$-GT to remove the glutamyl moiety from the glutathione conjugate to form EXE-cysteinylglycine or $17 \beta$-DHEcysteinylglycine, and a final reaction where the glycyl moiety is removed by dipeptidase to form the EXE-cys or $17 \beta$-DHE-cys conjugates.
In addition to the identified 6-EXE-cys (peak 1 in Fig. 2D) and 6-17 $\beta$ DHE-cys conjugates (peaks 3 in Fig. 3B), secondary peaks likely corresponding to EXE-cys (peak 2 in Fig. 2D) and 17 $\beta$-DHE-cys isomers (peaks 4 in Fig. 3B) of unknown structure were observed in the urine of women taking EXE in the present study. EXE and 17 $\beta$-DHE are $\alpha-\beta$ unsaturated ketones with several double bonds for GSH

TABLE 2

Summary of EXE metabolite concentrations in plasma and urine from EXE-treated subjects $(n=132$ subjects taking EXE)

\begin{tabular}{|c|c|c|c|c|c|c|}
\hline & \multicolumn{3}{|c|}{ Plasma } & \multicolumn{3}{|c|}{ Urine } \\
\hline & Mean \pm S.E. & Range & Percentage of TEM (Mean \pm S.E. $)^{a}$ & Mean \pm S.E. & Range & Percentage of TEM $(\text { Mean } \pm \text { S.E. })^{a}$ \\
\hline & $n M$ & & & nmol/mg creatinine & & \\
\hline EXE & $14 \pm 1.7$ & $0-105$ & $17 \pm 0.91$ & $0.21 \pm 0.045$ & $0-3.2$ & $1.7 \pm 0.20$ \\
\hline $17 \beta$-DHE & $2.5 \pm 0.19$ & $0.11-14$ & $12 \pm 1.2$ & $0.0066 \pm 0.0012$ & $0-0.099$ & $0.14 \pm 0.025$ \\
\hline $17 \beta$-DHE-Gluc & $30 \pm 4.6$ & $0.32-358$ & $36 \pm 1.8$ & $1.4 \pm 0.37$ & $0.0068-45$ & $21 \pm 1.6$ \\
\hline 6-EXE-cys & $22 \pm 2.9$ & $0.020-226$ & $23 \pm 1.3$ & $5.9 \pm 0.69$ & $0.0033-50$ & $55 \pm 1.6$ \\
\hline 6-17 $\beta$-DHE-cys & $5.9 \pm 0.58$ & $0-31$ & $12 \pm 0.76$ & $1.8 \pm 0.19$ & $0.0033-10$ & $22 \pm 0.79$ \\
\hline
\end{tabular}

${ }^{a} \mathrm{TEM}=\mathrm{EXE}+17 \beta$-DHE $+17 \beta$-DHE-Gluc +6 -EXE-cys +6 -DHE-cys. The percentage of TEM was calculated for EXE or each EXE metabolite for every individual subject, with the mean then calculated for each metabolite of all 132 subjects. 
<smiles>C[C@]12CC[C@H]3[C@@H](CC(CSC[C@H](N)C(=O)O)C4=CC(=O)C=C[C@]43C)[C@@H]1CCC2=O</smiles>

6-methylcysteinylandrosta1,4-diene-3-17-dione (6-EXE-cys)<smiles>C[C@]12C=CC(=O)C=C1C(CSC[C@H](N)C(=O)O)C[C@H]1[C@@H]2CC[C@]2(C)[C@@H](O)CC[C@@H]12</smiles>

6-methylcysteinylandrosta-1,4diene-17ß-hydroxy-3-one (6-17ß-DHE-cys)<smiles>C=C1C[C@]2(C)C3CCC(=O)[C@@]3(C)CCC2[C@]2(C)C=C[C@H](O)C[C@H]12</smiles>

$3 \xi$-hydroxy-5$\xi$-androsta-1-ene6-methylene-17-one

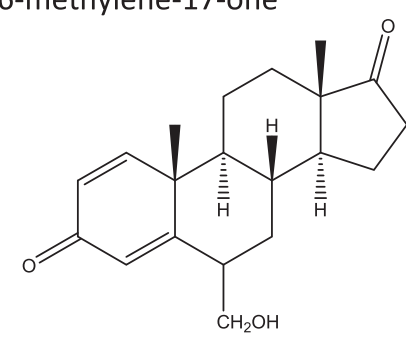
6-hydroxymethylexemestane

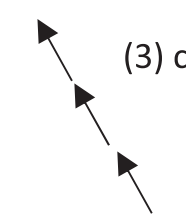

(3) cysteinylglycine dipeptidase

(2) $\gamma$-glutamyl transferase

(1) glutathione-S-transferase

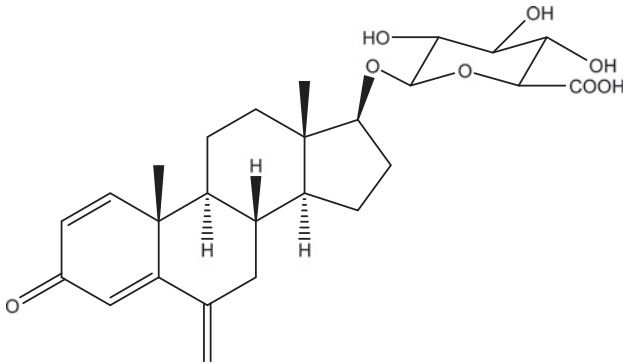

$17 \beta$-hydroxy-exemestane-17-O- $\beta$-Dglucuronide (17 $\beta$-DHE-Gluc)<smiles>C=C1C[C@]2(C)[C@@H]3CCC(=O)[C@@]3(C)CC[C@H]2[C@@]2(C)C=CC(=O)C=C12</smiles><smiles>CC1C[C@]2(C)[C@@H]3CC[C@@H](O)[C@@]3(C)CC[C@H]2[C@@]2(C)C=CC(=O)C=C12</smiles>

17 $\beta$-dihydro-exemestane (17 $\beta$-DHE) exemestane (EXE)

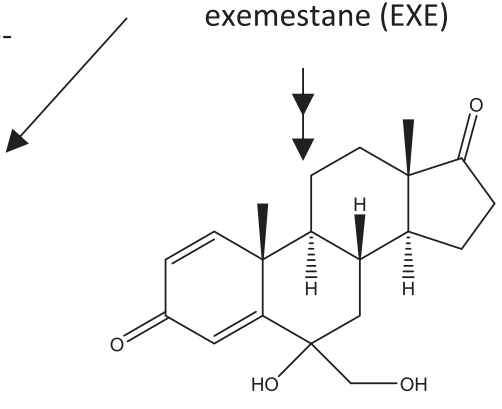

$6 \xi$-hydroxy- $6 \xi$-hydroxymethylandrosta1,4-diene-3,17-dione

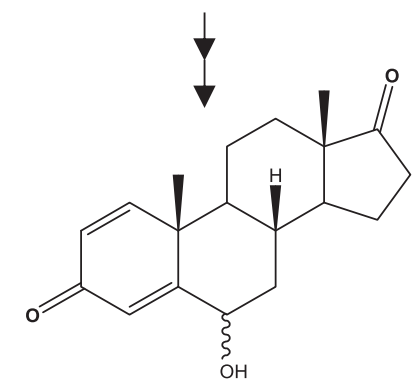<smiles>C[C@@]12C=CC(=O)C=C1C(O)(CO)C[C@@]1(C)[C@H]2CC[C@]2(C)[C@@H](O)CC[C@@H]21</smiles>

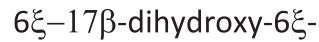
hydroxymethylandrosta-1,4-diene-3-one<smiles>C[C@]12CC[C@H]3[C@@]4(C)C=CC(=O)C=C4[C@H](O)C[C@@]3(C)C1CC[C@@H]2O</smiles>

$6 \xi$-hydroxyandrosta-1,4-diene-3,17-dione $6 \xi$-17 $\beta$-dihydroxyandrosta-1,4-diene-3-one

Scheme 1. Schematic of EXE metabolism. Shown are major exemestane metabolites formed in vivo.

conjugation. Therefore, there are several possible positions for the initial GSH conjugation step to occur. Chemical synthesis of other potential isomers of EXE-cys and $17 \beta$-DHE-cys conjugates is currently underway to confirm their structures.

Similar to phase II metabolism by glucuronidation, GSH conjugation of either EXE or 17 $\beta$-DHE likely increases their rate of excretion and eliminates their antiaromatase activities. Significant variations in levels of 6-EXE-cys and 6-17 $\beta$-DHE-cys were observed in the urine and plasma samples of the 132 subjects. As shown in Table 2, 6-EXE-cys and 6-17 $\beta$-DHE-cys ranged from 0.020 to $226 \mathrm{nM}$ and 0 to $31 \mathrm{nM}$ in plasma, respectively, and from 0.0033 to $50 \mathrm{nM}$ and 0.0033 to $10 \mathrm{nM}$ in urine, respectively. Interestingly, both the unidentified EXE cysteine conjugate isomer (peak 2 in Fig. 2D) and the unidentified 17 $\beta$-DHE cysteine conjugate isomer (peak 4 in Fig. 3B) were observed in the urine 
of only seven of the 10 subjects examined in this study. There is significant genetic variability in the GST enzymes (Hayes et al., 2005), including common copy number variants observed for both GSTM1 and GSTT1 (Seidegard et al., 1990; Bell et al., 1993; Arruda et al., 1998; Bailey et al., 1998; Roth et al., 2000; Hayes et al., 2005). The frequencies of homozygous deletion genotypes of GSTM1 and GSTT1 are about $50 \%$ and $14 \%$ in Caucasians, and the homozygous deletion genotypes of both GSTM1 and GSTT1 were reported to be associated with risk for a variety of cancers and may be linked to alterations in drug metabolism (Hengstler et al., 1998). For example, GSTs are involved in the metabolism of azathioprine (AZA) to mercaptopurine, with 6-methylmercaptopurine riboside a major metabolite of mercaptopurine. The levels of 6-methylmercaptopurine riboside were 2-fold lower in AZA users exhibiting the GSTM1-null genotype than AZA users carrying one or two copies of GSTM1 (Broekman et al., 2018). The deletion of GSTM1 was also associated with reduced response to AZA therapy (Stocco et al., 2014). This suggests that if such GSTs are similarly involved in the metabolism of EXE, copy number variants or other functional polymorphisms could potentially play an important role in the metabolism and efficacy of EXE. Although functional polymorphisms in both the $\gamma$-GT and dipeptidase enzymes could similarly modify the levels of EXE-cys or 17 $\beta$-DHE-cys observed in the plasma and/or urine of subjects taking EXE, they do not metabolize the functional parent compound, EXE, or its major active metabolite, $17 \beta$-DHE, and are, therefore, less likely to be important in overall patient response to EXE.

As described earlier, quantification of EXE-cys and 17 $\beta$-DHE-cys conjugates was performed only for peaks corresponding to the known 6-cysteine conjugates (peaks 1 and 3 in Figs. 2D and 3B, respectively). Although their structure is presently unknown, other peaks corresponding to other EXE-cys and 17 $\beta$-DHE-cys isomers were identified in the present study, potentially at levels similar to or higher than those observed for 6-EXE-cys and 6-17 $\beta$-DHE-cys. Therefore, these conjugates likely comprise an even larger proportion of EXE metabolites in the urine and plasma of women taking EXE, further increasing the importance of cysteine conjugate formation and decreasing the overall importance of glucuronidation in the metabolism of EXE. In addition, this further supports previous results demonstrating only small changes in plasma $17 \beta$-DHE and no change in urinary $17 \beta$-DHE in subjects deficient in DHE glucuronidation capacity since the glucuronide comprises a relatively low percentage of EXE metabolites in vivo (Luo et al., 2018).

A potential limitation of the present study was that the study subjects examined were primarily Caucasians (only five non-Caucasians out of 132 female subjects). Although we did not find observable differences in the levels of EXE or its metabolites between the Caucasian subjects and the five non-Caucasian subjects examined in this study, it is possible that the patterns observed for Caucasian females may not be $100 \%$ generalizable to all populations. Additional studies of EXE metabolism in other racial groups will be necessary to better examine this. Another potential limitation of the current study was that the intermediate products of the first and second steps for the proposed three-step pathway for EXE-cys and 17 $\beta$-DHE-cys formation were not detected in urine samples, suggesting that they are at low levels if present. This also suggests that the final two intermediate enzymatic reaction steps with $\gamma$-glutamyl transferase and cysteinylglycine dipeptidase to form the EXE-cys and $17 \beta$-DHE-cys conjugates are highly efficient.

In conclusion, two novel EXE phase II metabolites were identified in vivo in women taking EXE. The two metabolites-cysteine conjugates of EXE and 17 $\beta$-DHE - are the major metabolites of EXE found in the urine of subjects taking EXE, comprising, on average, 77\% of total quantified urinary EXE metabolites, and were at levels similar to that observed for the other major EXE metabolite, 17 $\beta$-DHE-Gluc, in the plasma of the same subjects. Although their exact structure is presently unknown, other EXE and $17 \beta$-DHE cysteine conjugates were also identified, further supporting cysteine conjugate formation as the major metabolism pathway for EXE in vivo. The pathways involved in EXE-cys or 17 $\beta$-DHE-cys formation could, therefore, potentially play an important role in the pharmacokinetics and pharmacodynamics of EXE.

\section{Acknowledgments}

We thank the Mass Spectrometry Core facility and the Nuclear Magnetic Resonance Core facility at Washington State University Spokane for their help with UPLC-MS and NMR, respectively.

\section{Authorship Contributions}

Participated in research design: Luo, Chen, Truica, Lazarus.

Conducted experiments: Luo, Chen, Baird, Xia.

Performed data analysis: Luo, Chen, Xia, Lazarus.

Wrote or contributed to the writing of the manuscript: Luo, Chen, Truica, Xia, Lazarus.

\section{References}

Arruda VR, Grignolli CE, Gonçalves MS, Soares MC, Menezes R, Saad ST, and Costa FF (1998) Prevalence of homozygosity for the deleted alleles of glutathione S-transferase mu (GSTM1) and theta (GSTT1) among distinct ethnic groups from Brazil: relevance to environmental carcinogenesis? Clin Genet 54:210-214.

Ashmore JH, Lesko SM, Muscat JE, Gallagher CJ, Berg AS, Miller PE, Hartman TJ, and Lazarus P (2013) Association of dietary and supplemental folate intake and polymorphisms in three FOCM pathway genes with colorectal cancer in a population-based case-control study. Genes Chromosomes Cancer 52:945-953.

Bailey LR, Roodi N, Verrier CS, Yee CJ, Dupont WD, and Parl FF (1998) Breast cancer and CYPIA1, GSTM1, and GSTT1 polymorphisms: evidence of a lack of association in Caucasians and African Americans. Cancer Res 58:65-70.

Bateman KP, Castro-Perez J, Wrona M, Shockcor JP, Yu K, Oballa R, and Nicoll-Griffith DA (2007) MSE with mass defect filtering for in vitro and in vivo metabolite identification. Rapid Commun Mass Spectrom 21:1485-1496.

Bell DA, Taylor JA, Paulson DF, Robertson CN, Mohler JL, and Lucier GW (1993) Genetic risk and carcinogen exposure: a common inherited defect of the carcinogen-metabolism gene glutathione S-transferase M1 (GSTM1) that increases susceptibility to bladder cancer. J Natl Cancer Inst 85:1159-1164.

Broekman MMTJ, Wong DR, Wanten GJA, Roelofs HM, van Marrewijk CJ, Klungel OH, Verbeek ALM, Hooymans PM, Guchelaar HJ, Scheffer H, et al. (2018) The glutathione transferase Mu null genotype leads to lower 6-MMPR levels in patients treated with azathioprine but not with mercaptopurine. Pharmacogenomics J 18:160-166.

Campos SM (2004) Aromatase inhibitors for breast cancer in postmenopausal women. Oncologist 9:126-136.

Campos SM, Guastalla JP, Subar M, Abreu P, Winer EP, and Cameron DA (2009) A comparative study of exemestane versus anastrozole in patients with postmenopausal breast cancer with visceral metastases. Clin Breast Cancer 9:39-44.

Cappiello M, Lazzarotti A, Buono F, Scaloni A, D’Ambrosio C, Amodeo P, Méndez BL, Pelosi P, Del Corso A, and Mura U (2004) New role for leucyl aminopeptidase in glutathione turnover. Biochem J 378:35-44.

Cavalcanti Gde A, Garrido BC, Leal FD, Padilha MC, de la Torre X, and de Aquino Neto FR (2011) Detection of new urinary exemestane metabolites by gas chromatography coupled to mass spectrometry. Steroids 76:1010-1015.

Chia S, Gradishar W, Mauriac L, Bines J, Amant F, Federico M, Fein L, Romieu G, Buzdar A, Robertson JF, et al. (2008) Double-blind, randomized placebo controlled trial of fulvestrant compared with exemestane after prior nonsteroidal aromatase inhibitor therapy in postmenopausal women with hormone receptor-positive, advanced breast cancer: results from EFECT. J Clin Oncol 26:1664-1670.

Chin CC and Warren JC (1972) Synthesis of 2 -, 6 -, and 6 -bromoprogesterone and study of the binding site of 20 -hydroxysteroid dehydrogenase. Biochemistry 11:2720-2726.

de Albuquerque Cavalcanti G, Carius Garrido B, Dias Leal F, Costa Padilha M, Mazzarino M, de la Torre X, Botre F, and Radler de Aquino Neto F (2011) Detection of new exemestane metabolites by liquid chromatography interfaced to electrospray-tandem mass spectrometry. I Steroid Biochem Mol Biol 127:248-254.

Deeks ED and Scott LJ (2009) Exemestane: a review of its use in postmenopausal women with breast cancer. Drugs 69:889-918.

Del Corso A, Cappiello M, Buono F, Moschini R, Paolicchi A, and Mura U (2006) Colorimetric coupled enzyme assay for gamma-glutamyltransferase activity using glutathione as substrate. $J$ Biochem Biophys Methods 67:123-130.

Eisen A, Trudeau M, Shelley W, Messersmith H, and Pritchard KI (2008) Aromatase inhibitors in adjuvant therapy for hormone receptor positive breast cancer: a systematic review. Cancer Treat Rev 34:157-174.

Evans TR, Di Salle E, Ornati G, Lassus M, Benedetti MS, Pianezzola E, and Coombes RC (1992) Phase I and endocrine study of exemestane (FCE 24304), a new aromatase inhibitor, in postmenopausal women. Cancer Res 52:5933-5939.

Ferretti G, Bria E, Giannarelli D, Felici A, Papaldo P, Fabi A, Di Cosimo S, Ruggeri EM, Milella M, Ciccarese M, et al. (2006) Second- and third-generation aromatase inhibitors as first-line 
endocrine therapy in postmenopausal metastatic breast cancer patients: a pooled analysis of the randomised trials. Br J Cancer 94:1789-1796.

Forbes JF, Cuzick J, Buzdar A, Howell A, Tobias JS, and Baum M; Arimidex, Tamoxifen, Alone or in Combination (ATAC) Trialists' Group (2008) Effect of anastrozole and tamoxifen as adjuvant treatment for early-stage breast cancer: 100-month analysis of the ATAC trial. Lancet Oncol 9:45-53.

Glück S (2010) Exemestane as first-line therapy in postmenopausal women with recurrent or metastatic breast cancer. Am J Clin Oncol 33:314-319.

Görlitzer K, Bonnekessel Ch, Jones PG, Palusczak A, and Hartmann RW (2006) Derivatives of exemestane--synthesis and evaluation of aromatase inhibition. Pharmazie 61:575-581.

Grillo MP, Hua F, March KL, Benet LZ, Knutson CG, and Ware JA (2008) Gammaglutamyltranspeptidase-mediated degradation of diclofenac-S-acyl-glutathione in vitro and in vivo in rat. Chem Res Toxicol 21:1933-1938.

Hayes JD, Flanagan JU, and Jowsey IR (2005) Glutathione transferases. Annu Rev Pharmacol Toxicol 45:51-88.

Hengstler JG, Arand M, Herrero ME, and Oesch F (1998) Polymorphisms of N-acetyltransferases, glutathione S-transferases, microsomal epoxide hydrolase and sulfotransferases: influence on cancer susceptibility. Recent Results Cancer Res 154:47-85.

Hinchman CA and Ballatori N (1994) Glutathione conjugation and conversion to mercapturic acids can occur as an intrahepatic process. J Toxicol Environ Health 41:387-409.

Hirota T, Nishikawa Y, Tanaka M, Igarashi T, and Kitagawa H (1986) Characterization of dehydropeptidase I in the rat lung. Eur J Biochem 160:521-525.

Hong Y, Yu B, Sherman M, Yuan YC, Zhou D, and Chen S (2007) Molecular basis for the aromatization reaction and exemestane-mediated irreversible inhibition of human aromatase. $\mathrm{Mol}$ Endocrinol 21:401-414.

Howell A, Cuzick J, Baum M, Buzdar A, Dowsett M, Forbes JF, Hoctin-Boes G, Houghton J, Locker GY, and Tobias JS; ATAC Trialists' Group (2005) Results of the ATAC (Arimidex, Tamoxifen, Alone or in Combination) trial after completion of 5 years' adjuvant treatment for breast cancer. Lancet $\mathbf{3 6 5}: 60-62$.

Howlader N, Noone AM, Krapcho M, Miller D, Bishop K, Altekruse SF, Kosary CL, Yu M, Ruhl J, Tatalovich Z, et al. SEER Cancer Statistics Review, 1975-2013.

National Cancer Institute. Bethesda, MD, https://seer.cancer.gov/archive/csr/1975_2013/, based on November 2015 SEER data submission, posted to the SEER web site, April 2016.

Jösch C, Sies H, and Akerboom TP (1998) Hepatic mercapturic acid formation: involvement of cytosolic cysteinylglycine S-conjugate dipeptidase activity. Biochem Pharmacol 56:763-771.

Kamdem LK, Flockhart DA, and Desta Z (2011) In vitro cytochrome P450-mediated metabolism of exemestane. Drug Metab Dispos 39:98-105.

Lash LH, Lipscomb JC, Putt DA, and Parker JC (1999) Glutathione conjugation of trichloroethylene in human liver and kidney: kinetics and individual variation. Drug Metab Dispos 27:351-359

Luo S, Chen G, Truica C, Baird CC, Leitzel K, and Lazarus P (2018) Role of the UGT2B17 deletion in exemestane pharmacogenetics. Pharmacogenomics $J$ 18:295-300.

Miller WR (1999) Biology of aromatase inhibitors: pharmacology/endocrinology within the breast. Endocr Relat Cancer 6:187-195.

Numazawa M and Oshibe M (1994) 6-Alkyl- and 6-arylandrost-4-ene-3,17-diones as aromatase inhibitors. Synthesis and structure-activity relationships. J Med Chem 37:1312-1319.

Numazawa M and Yamaguchi S (1998) 6-Phenylaliphatic-substituted androst-4-ene-3,17-diones as aromatase inhibitors: structure-activity relationships. J Steroid Biochem Mol Biol 67:41-48.

Osborne CK and Schiff R (2011) Mechanisms of endocrine resistance in breast cancer. Annu Rev Med 62:233-247.
Paridaens R, Dirix L, Lohrisch C, Beex L, Nooij M, Cameron D, Biganzoli L, Cufer T, Duchateau L, Hamilton A, et al.; European Organization for the Research and Treatment of Cance (EORTC)- Investigational Drug Branch for Breast Cancer (IDBBC) (2003) Mature results of a randomized phase II multicenter study of exemestane versus tamoxifen as first-line hormone therapy for postmenopausal women with metastatic breast cancer. Ann Oncol 14:1391-1398.

Peterson A, Xia Z, Chen G, and Lazarus P (2017) In vitro metabolism of exemestane by hepatic cytochrome P450s: impact of nonsynonymous polymorphisms on formation of the active metabolite 17 $\beta$-dihydroexemestane. Pharmacol Res Perspect 5:e0314.

Petkov PI, Temelkov S, Villeneuve DL, Ankley GT, and Mekenyan OG (2009) Mechanism-based categorization of aromatase inhibitors: a potential discovery and screening tool. SAR QSAR Environ Res 20:657-678.

Platt A, Xia Z, Liu Y, Chen G, and Lazarus P (2016) Impact of nonsynonymous single nucleotide polymorphisms on in-vitro metabolism of exemestane by hepatic cytosolic reductases. Phar macogenet Genomics 26:370-380.

Plumb RS, Johnson KA, Rainville P, Smith BW, Wilson ID, Castro-Perez JM, and Nicholson JK (2006) UPLC/MS(E); a new approach for generating molecular fragment information for biomarker structure elucidation. Rapid Commun Mass Spectrom 20:1989-1994.

Roth MJ, Dawsey SM, Wang G, Tangrea JA, Zhou B, Ratnasinghe D, Woodson KG, Olivero OA, Poirier MC, Frye BL, et al. (2000) Association between GSTM1*0 and squamous dysplasia of the esophagus in the high risk region of Linxian, China. Cancer Lett 156:73-81.

Santen RJ, Brodie H, Simpson ER, Siiteri PK, and Brodie A (2009) History of aromatase: saga of an important biological mediator and therapeutic target. Endocr Rev 30:343-375.

Schneider F, Boller A, Müller M, Müller P, and Fürst A (1973) The course of the transformation of steroid 3,5-dienamines with formaldehyde. Helv Chim Acta 56:2396-2404.

Seidegård J, Pero RW, Markowitz MM, Roush G, Miller DG, and Beattie EJ (1990) Isoenzyme(s) of glutathione transferase (class $\mathrm{Mu}$ ) as a marker for the susceptibility to lung cancer: a follow up study. Carcinogenesis 11:33-36.

Shi J, Xie C, Liu H, Krausz KW, Bewley CA, Zhang S, Tang L, Zhou Z, and Gonzalez FJ (2016) Metabolism and bioactivation of fluorochloridone, a novel selective herbicide, in vivo and in vitro. Environ Sci Technol 50:9652-9660.

Siegel RL, Miller KD, and Jemal A (2017) Cancer statistics, 2017. CA Cancer J Clin 67:7-30.

Stocco G, Cuzzoni E, De Iudicibus S, Franca R, Favretto D, Malusà N, Londero M, Cont G, Bartol F, Martelossi S, et al (2014) Deletion of glutathione-s-transferase $\mathrm{ml}$ reduces azathioprine metabolite concentrations in young patients with inflammatory bowel disease. J Clin Gastroenterol 48:43-51.

Sun D, Chen G, Dellinger RW, Sharma AK, and Lazarus P (2010) Characterization of 17-dihydroexemestane glucuronidation: potential role of the UGT2B17 deletion in exemestane pharmacogenetics. Pharmacogenet Genomics 20:575-585.

van Bladeren PJ (2000) Glutathione conjugation as a bioactivation reaction. Chem Biol Interact 129:61-76.

Wang X and Chen S (2006) Aromatase destabilizer: novel action of exemestane, a food and drug administration-approved aromatase inhibitor. Cancer Res 66:10281-10286.

Zarth AT, Murphy SE, and Hecht SS (2015) Benzene oxide is a substrate for glutathione S-transferases. Chem Biol Interact 242:390-395.

Address correspondence to: Philip Lazarus, College of Pharmacy and Pharmaceutical Sciences, Washington State University, PBS Building Room 431, P.O. Box 1495, Spokane, WA 99202. E-mail: phil.lazarus@wsu.edu 